\title{
The composite-spectrum binary hypothesis does not explain the $\lambda$ Bootis stars
}

\author{
R. E. Griffin ${ }^{1, \star}$, R. O. Gray ${ }^{2}$, and C. J. Corbally ${ }^{3}$ \\ ${ }^{1}$ Dominion Astrophysical Observatory, 5071 West Saanich Road, Victoria, BC, V9E 2E7, Canada \\ e-mail: Elizabeth.Griffin@nrc.gc.ca \\ 2 Department of Physics and Astronomy, Appalachian State University, Boone, NC 28608, USA \\ e-mail: Grayro@appstate.edu \\ 3 Vatican Observatory Research Group, Steward Observatory, Tucson, AZ 85721-0065, USA \\ e-mail: Corbally@as.arizona.edu
}

Received 24 February 2012 / Accepted 17 June 2012

\begin{abstract}
The existence of the $\lambda$ Boo type as a class of chemically-peculiar stars in its own right has taxed numerous researchers, and has challenged spectroscopists to produce a model which is plausible, comprehensive and predictive. Stars which are recognized as members of the $\lambda$ Boo class have late-B to early-F spectral types, and exhibit (often substantially) low abundances of Fe-peak elements although elements such as C, N, O and S may have more nearly solar abundances. Since less than $2 \%$ of objects within the relevant spectral-type domain appear to be $\lambda$ Boo stars their existence has demanded rather special conditions, and has triggered opinion that this group may not in fact exist but that each case can be explained as an unrecognized binary. In this paper we examine those claims by monitoring 10 stars, listed in the literature as possible $\lambda$ Boo stars but said to be "likely candidates" for composite-spectrum binaries, by employing high-dispersion spectroscopy in an intermittent observing programme designed to reveal the sort of line-profile changes that should be detectable if each object were really a pair of similar stars in an SB2 system. We also monitor two other stars: HR 7903, said to be a binary (but is more like an Ap star), and $\lambda$ Boo itself. The sample includes 1 possible, 1 marginal and 4 definite $\lambda$ Boo classifications. In addition, we derive the physical properties of the 12 stars by photometric and spectroscopic synthesis, and measure their radial velocities. Three of the sample show small line-profile variations, but not of the sort that can be attributed to the presence of a companion star; they are the suspected Ap star HR 7903, HR 6878 (which exhibits spectrum peculiarities very similar to those of HR 7903 but has not previously been classified as Bp or Ap), and $\lambda$ Aql, whose rapid spectrum variations resemble those observed in spotted or CP stars. None of the stars shows any evidence to suggest that it could be a composite-spectrum binary.
\end{abstract}

Key words. stars: early-type - binaries: spectroscopic - stars: chemically peculiar - stars: individual: $\lambda$ Bootis

\section{Background}

The $\lambda$ Boo stars constitute an elite group of late-B to early-F stars that are decidedly weak in Fe-peak elements $(\lambda$ Boo itself has a metallicity $[\mathrm{M} / \mathrm{H}]$ of about -2 dex) but are only slightly weak, if at all, in $\mathrm{C}, \mathrm{N}, \mathrm{O}$ and $\mathrm{S}$. In general the spectral lines are broadened by rotation of the order of $100-150 \mathrm{~km} \mathrm{~s}^{-1}$. As a group, the $\lambda$ Boo stars lie close to the pulsation instability strip in the HR diagram, and a significant fraction have been found to exhibit non-radial pulsations (Bohlender et al. 1999). Those and other characteristics of the group have been described eloquently and comprehensively elsewhere, e.g. Paunzen (2001), Bohlender et al. (1999) and in papers cited therein.

Less than $2 \%$ of all stars in that temperature range appear to be $\lambda$ Boo stars, thus demanding either a very short time-scale (maybe 1 million years) during which the phenomenon can occur, or somewhat rare conditions for it to happen at all. That need to accept what may be a rather special set of conditions for this sub-set of chemically-peculiar A stars has triggered alternative suggestions that they may not in fact be a separate group, but that each individual object which has been so classified can be explained by binarity or by misclassification. Those opinions

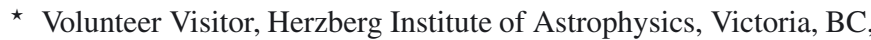
Canada. have been expressed most strongly by Faraggiana \& Bonifacio (1999), Gerbaldi et al. (2003, hereafter G+2003), Faraggiana et al. (2004, hereafter F+2004), and in earlier papers by that group and quoted by them. The main focus of their suggestions was the possibility that $\lambda$ Boo stars are unrecognised binaries consisting of two fairly similar stars, so the apparently single spectrum which is observed is in reality composite, the continuum flux of one component weakening the absorption lines of its companion. In their own words, F+2004 assert that "some of the underabundances reported in the literature may simply be the result of the failure to consider the composite nature of the spectra. This leads to the legitimate suspicion that some, if not all, the $\lambda$ Boo candidates are not chemically peculiar at all". The blurring caused by the rotational broadening of both stars would presumably have to be sufficient to mask their identification as double-lined spectroscopic binaries (SB2).

On the surface Faraggiana, Gerbaldi and co-authors appear to have a point. It is not easy to distinguish between genuine line-doubling and rotational broadening in the spectrum of an early-type star if its rotational velocity is high, and numerous entries of "V?" in the Bright Star Catalogue (BSC) (Hoffleit 1982) for early-type stars can be attributed to that uncertainty. In their Table $1 \mathrm{G}+2003$ assembled 136 entries from assorted tabulations of suggested candidate $\lambda$ Boo objects, and proceeded to show that in many cases the special characteristics which 
Table 1. The sample of 12 stars.

\begin{tabular}{|c|c|c|c|c|c|c|}
\hline Star & HR & HD & $\begin{array}{c}V \\
(\mathrm{mag})\end{array}$ & $\begin{array}{c}(B-V) \\
(\mathrm{mag})\end{array}$ & Spectral type* & $\begin{array}{l}\text { Prime reason(s) for } \\
\text { inclusion in } G+2003\end{array}$ \\
\hline \multirow[t]{2}{*}{$21 \mathrm{LMi}$} & 3974 & 87696 & 4.7 & 0.18 & $\mathrm{~A} 7 \mathrm{~V}(\mathrm{n})$ & $\mathrm{AM}^{\dagger}$; inconsistent UV flux \\
\hline & 4875 & 111604 & 6.0 & 0.15 & A8 Vn kA1.5 mA1.5 $\lambda$ Boo & $\mathrm{AM}$; variable RV \\
\hline \multirow[t]{2}{*}{$\lambda \mathrm{Boo}$} & 5351 & 125162 & 3.2 & 0.08 & A3 Va kB9mB9 $\lambda$ Boo & Gray classification \\
\hline & 5368 & 125489 & 6.4 & 0.20 & A7 Vn & AM; inconsistent UV flux \\
\hline \multirow[t]{2}{*}{$12 \mathrm{Ser}$} & 5770 & 138527 & 6.2 & -0.04 & B9 IV ( $\lambda$ Boo $)$ & AM; HIPPARCOS double \\
\hline & 6878 & 169009 & 6.5 & 0.12 & B9 V He wk (CP) & AM; variable RV \\
\hline \multirow[t]{2}{*}{$\lambda \mathrm{Aql}$} & 7236 & 177756 & 3.4 & -0.09 & B9 IV (He wk) (CP) & $\mathrm{AM}$; variable RV \\
\hline & 7288 & 179791 & 6.6 & 0.09 & A $1.5 \mathrm{IVn}$ & Inconsistent UV flux \\
\hline \multirow[t]{2}{*}{35 Aql } & 7400 & 183324 & 5.8 & 0.08 & $\mathrm{~A} 0 \mathrm{Vb} \lambda \mathrm{Boo}$ & Gray classification \\
\hline & 7903 & 196821 & 6.1 & -0.05 & A0 $\mathrm{III}^{+} \mathrm{p}$ kB8 mA1 (CP) & AM; UV flux \\
\hline$\tau$ Peg & 8880 & 220061 & 4.8 & 0.17 & A8 V(n) kA5mA5 ( $\lambda$ Boo) & $\mathrm{AM}$; variable RV \\
\hline 15 And & 8947 & 221756 & 5.6 & 0.10 & $\mathrm{~A} 1 \mathrm{Va} \lambda \mathrm{Boo}$ & Gray classification \\
\hline
\end{tabular}

Notes. ( $\lambda$ Boo) indicates marginal classification. ${ }^{(\dagger)}$ Included in the list of Abt \& Morrell (1995). ${ }^{(*)}$ This paper, except for $\lambda$ Boo (Gray et al. 2003).

had earned inclusion in that list could in fact be explained through unrecognized duplicity, taking as their evidence variable RV, photometry (especially raised UV flux), astrometric measurements or possible binarity recorded in the HIPPARCOS catalogue (ESA 1997). Others could be accounted for as misclassifications. An insufficiently founded assignment of "V" or "V?" in the BSC has therefore played a rôle here. However, $\mathrm{G}+2003$ did not take into account the filtering which had already been carried out elsewhere in order to produce a definitive list of accredited $\lambda$ Boo stars, so most (if not all) of the stars which they rejected had already been rejected by others. The high rejection rate which $\mathrm{G}+2003$ thus obtained as due to unrecognized binarity and which they then extrapolated to the remainder of the sample is therefore not valid evidence in their argument.

On the strength of the statistics of the high incidence of SB systems in their initial list of 136 possible $\lambda$ Boo stars, F+2004 adopted a composite-spectrum explanation as their working hypothesis. From their initial list they filtered out 12 objects which appeared to be astrometric binaries, and a further 10 which were confirmed as, or which they had shown from high-resolution spectroscopy to be, binaries (one such was HD $196821=$ HR 7903, which in fact, as we show below, is not a spectroscopic binary). They then prioritized 14 other stars which they rated as "likely" to display composite spectra, having "variable RV and not belonging to known visual or spectroscopic binary systems", stating that "the most urgent thing ... is to perform ... a detailed analysis for as many $\lambda$ Boo stars with composite spectra as possible. It is of paramount importance that such an analysis is based on many spectra, taken at different phases, with as large a spectral coverage as possible". We took up that challenge, and this paper describes the results of our efforts. If the compositespectrum hypothesis is tenable and the statistical arguments of F+2004 hold, then their sub-set of 14 should reveal binarity unequivocally through appropriate spectroscopy. We therefore adopted it as the foundation for this paper.

Since the project commenced in summertime, we excluded from F+2004's sample of 14 three winter-time objects and one that was rather far south. To the remaining 10 objects we added $\lambda$ Boo itself, and HR 7903. The latter was chosen from F+2004's list of "confirmed" binaries because those authors had concluded, from a discussion of asymmetries in its lines based on a single observation, that it is a composite-spectrum SB2 system; they could reject it as a $\lambda$ Boo candidate according to a number of criteria, but had included it in their initial list of candidates because its identity as a possible $\lambda$ Boo star had been suggested by Abt \& Morrell (1995) - a paper which constituted a major source for their study. (However, papers published since have shown that a number of the $\lambda$ Boo classifications listed by Abt \& Morrell cannot be upheld.) What we were therefore testing in the case of HR 7903 was its status as a "confirmed" SB2 system and the ability to recognize it as such from a single observation.

We have reviewed and in some cases revised the classifications of the 12 stars in our sample. Of those, four are confirmed $\lambda$ Boo stars, one is tentatively so classified and one is a marginal $\lambda$ Boo star. Half of the sample stars are therefore definite or probable $\lambda$ Boo objects (see Table 1). Table 1 also gives the prime reason(s) why each star was included in G+2003's initial list (their Table 1) in the first place. We begin by describing the observational material that was the core of our work (Sect. 2), and illustrate it in Sect. 3 to demonstrate the paper's conclusions. In Sect. 4 we back up those conclusions with radial-velocity (RV) measurements that we made on all the spectra. However, rather than leave the matter at a rather negative conclusion, we go on to extract the physical parameters of the stars through flux modelling and synthetic-spectrum fitting (Sect. 5). In Sect. 6 we examine sharp features ("pips") which appear in the Ca II K-line cores of half of our sample, and attempt to distinguish between a circumstellar (CS) and an interstellar (IS) origin. In Sect. 7 we give some notes on each individual star. Finally, in Sect. 8 we amplify our conclusion that the stars proposed by $\mathrm{G}+2003$ as "likely composite-spectrum candidates" are not spectroscopic binaries, and that the $\lambda$ Boo phenomenon cannot be dismissed by that route.

\section{Observations}

High-dispersion, high-resolution, high-S/N spectra $(2.2 \AA / \mathrm{mm}$, $R \approx 45000$ ) were recorded on a SITe-4 CCD at the coudé focus of the 96-inch spectrograph of the Dominion Astrophysical Observatory (DAO)'s 1.2-m telescope. Each spectrum spans approximately $145 \AA$. Exposure times were mostly one hour (sometimes less) in order to achieve $S / N>100$, but when conditions so dictated, two spectra were observed sequentially and coadded in order to improve the $\mathrm{S} / \mathrm{N}$. The observations were stored as FITS files, and reduced to 1-D spectra in the heliocentricvelocity rest-frame by a semi-automatic pipeline. A correction of $3.5 \%$ for white-light scattering was applied.

Our initial plan was to observe each star at two wavelength regions - one centred on the $\mathrm{Ca}$ II $\mathrm{K}$ line and the other on the 
Table 2. Log of spectroscopic observations of the 12 candidate stars.

\begin{tabular}{|c|c|c|c|c|c|c|c|c|c|c|c|}
\hline UT date & $\lambda_{\mathrm{c}}$ & File & $S / N$ & UT date & $\lambda_{\mathrm{c}}$ & File & $S / N$ & UT date & $\lambda_{\mathrm{c}}$ & File & $S / N$ \\
\hline $21 \mathrm{LMi}$ & & & & 12 Ser* & & & & $35 \mathrm{Aql}^{*}$ & & & \\
\hline 2001 Feb. 19.24 & 3933 & 1434 & 105 & 2005 Apr. 19.43 & 3933 & 5375 & 150 & 2004 Jul. 19.37 & 4481 & 13526 & 155 \\
\hline 2001 Feb. 20.29 & 3933 & 1498 & 150 & 2005 Apr. 22.35 & 4481 & 5529 & 170 & 2004 Jul. 22.26 & 3933 & 13674 & 140 \\
\hline 2005 Apr. 19.26 & 3933 & 5371 & 240 & 2007 May 23.33 & 4481 & 6144 & 240 & 2004 Sep. 19.21 & 3933 & 20137 & 130 \\
\hline 2005 Apr. 23.26 & 3933 & 5578 & 255 & 2008 Apr. 9.46 & 4481 & 4685 & 130 & 2004 Sep. 20.18 & 3933 & 20183 & 140 \\
\hline 2005 Apr. 28.28 & 4481 & 5843 & 335 & 2008 May 5.42 & 4481 & 5593 & 145 & 2005 Aug. 24.25 & 4481 & 14716 & 220 \\
\hline 2007 Nov. 22.52 & 4481 & 19493 & 230 & 2008 May 17.44 & 4481 & 6486 & 155 & 2005 Aug. 26.27 & 4481 & 14828 & 300 \\
\hline 2007 Nov. 23.52 & 3933 & 19549 & 210 & 2008 Sep. 3.17 & 3933 & 13974 & 100 & 2007 Aug. 25.35 & 3933 & 11755 & 285 \\
\hline 2008 Jan. 15.43 & 4481 & 106 & 195 & 2008 Sep. 11.15 & 3933 & 15429 & 110 & 2008 Jun. 25.38 & 4481 & 8851 & 145 \\
\hline 2008 Jan. 20.48 & 4481 & $197 / 8$ & 190 & 2010 Mar. 23.47 & 3933 & $5713 / 4$ & 108 & 2008 Aug. 14.29 & 4481 & 13146 & 235 \\
\hline 2008 May 1.27 & 4481 & 5446 & 250 & 2010 May 17.37 & 3933 & 9880 & 114 & 2010 Jun. 12.37 & 3933 & 10502 & 135 \\
\hline HR $4875^{*}$ & & & & HR 6878 & & & & HR 7903 & & & \\
\hline 2005 Apr. 19.30 & 3933 & 5372 & 150 & 2005 Apr. 19.51 & 3933 & 5377 & 107 & 2002 Oct. 14.23 & 3933 & 18319 & 160 \\
\hline 2005 Apr. 23.30 & 3933 & 5579 & 135 & 2005 Apr. 22.48 & 4481 & 5532 & 105 & 2002 Oct. 16.27 & 4481 & 18451 & 170 \\
\hline 2007 Nov. 21.59 & 3933 & 19427 & 115 & 2005 Apr. 28.45 & 4481 & 5847 & 100 & 2004 Jul. 23.33 & 3933 & 13730 & 130 \\
\hline 2008 Jan. 15.52 & 4481 & 108 & 140 & 2005 Aug. 24.18 & 4481 & 14714 & 140 & 2004 Jul. 25.30 & 3933 & 13833 & 130 \\
\hline 2008 Apr. 9.34 & 4481 & 4682 & 180 & 2005 Aug. 26.23 & 4481 & 14827 & 155 & 2004 Sep. 20.21 & 3933 & 20184 & 145 \\
\hline 2008 May 1.32 & 4481 & 5447 & 100 & 2005 Sep. 1.17 & 3933 & 15156 & 130 & 2008 Aug. 14.34 & 4481 & 13147 & 175 \\
\hline 2008 May 5.28 & 4481 & $5589 / 0$ & 195 & 2007 May 24.36 & 4481 & 6206 & 125 & 2008 Aug. 15.36 & 4481 & 13205 & 165 \\
\hline 2008 May 17.29 & 4481 & 6482 & 160 & 2008 Sep. 11.19 & 3933 & 15430 & 105 & 2008 Aug. 16.29 & 4481 & 13257 & 180 \\
\hline 2010 Mar. 23.40 & 3933 & $5711 / 2$ & 119 & 2009 Jun. 3.33 & 3933 & 10949 & 105 & 2008 Sep. 12.28 & 3933 & 15484 & 140 \\
\hline 2010 May 17.20 & 3933 & 9876 & 115 & 2010 Jun. 12.41 & 3933 & $10452 / 4$ & 118 & 2008 Sep. 29.15 & 4481 & 16928 & 130 \\
\hline$\lambda$ Boo* $^{*}$ & & & & $\lambda \mathrm{Aql}$ & & & & $\tau$ Peg* $^{*}$ & & & \\
\hline 2008 May 4.44 & 3933 & 5542 & 160 & 2005 Apr. 20.47 & 3933 & 5429 & 374 & 2005 Aug. 25.49 & 4481 & 14779 & 185 \\
\hline 2008 May 5.38 & 4481 & 5592 & 240 & 2005 Apr. 21.51 & 3933 & 5481 & 403 & 2007 Aug. 24.52 & 3933 & 11708 & 190 \\
\hline 2008 May 17.32 & 4481 & 6483 & 155 & 2005 Apr. 26.41 & 3933 & 5722 & 261 & 2007 Aug. 25.39 & 3933 & 11756 & 190 \\
\hline 2008 May 18.34 & 3933 & 6533 & 140 & 2005 Aug. 21.25 & 4481 & 14542 & 427 & 2007 Nov. 22.25 & 4481 & 19486 & 240 \\
\hline 2008 Jun. 27.22 & 3933 & 8976 & 165 & 2005 Aug. 26.16 & 4481 & 14825 & 355 & 2007 Nov. 23.35 & 3933 & 19545 & 130 \\
\hline 2008 Jul. 24.25 & 3933 & 11871 & 180 & 2005 Sep. 1.34 & 4481 & 15175 & 399 & 2008 Jan. 20.14 & 4481 & $193 / 4$ & 140 \\
\hline 2008 Aug. 14.26 & 4481 & 13145 & 260 & 2007 May 22.50 & 3933 & 6098 & 173 & 2008 Jul. 24.48 & 3933 & 11877 & 130 \\
\hline 2008 Sep. 1.20 & 3933 & 13870 & 210 & 2007 May 23.42 & 4481 & 6146 & 307 & 2008 Aug. 15.48 & 4481 & 13208 & 210 \\
\hline 2008 Sep. 28.12 & 4481 & $16873 / 4$ & 245 & 2008 Aug. 16.22 & 4481 & 13255 & 255 & 2008 Aug. 16.45 & 4481 & 13261 & 210 \\
\hline 2008 Sep. 29.12 & 4481 & 16927 & 190 & 2009 Aug. 19.22 & 3933 & 19061 & 332 & 2008 Sep. 2.36 & 3933 & 13926 & 180 \\
\hline HR 5368 & & & & HR 7288 & & & & 15 And$^{*}$ & & & \\
\hline 2005 Apr. 19.34 & 3933 & 5373 & 126 & 2009 Aug. 16.29 & 3933 & 18906 & 145 & 2004 Jul. 20.37 & 4481 & 13577 & 104 \\
\hline 2007 May 22.34 & 3933 & 6094 & 110 & 2009 Aug. 18.30 & 3933 & $19007 / 8$ & 115 & 2004 Sep. 14.24 & 3933 & $19951 / 2$ & 150 \\
\hline 2008 Apr. $\quad 9.42$ & 4481 & 4684 & 135 & 2009 Aug. 22.22 & 3933 & $19331 / 2$ & 150 & 2004 Sep. 20.24 & 3933 & 20185 & 145 \\
\hline 2008 Jun. 27.24 & 3933 & 8977 & 105 & 2009 Aug. 31.19 & 3933 & 19708 & 135 & 2005 Jan. 5.21 & 3933 & 146 & 100 \\
\hline 2008 Aug. 14.23 & 4481 & 13144 & 108 & 2009 Sep. 20.18 & 3933 & $23133 / 4$ & 150 & 2005 Aug. 24.38 & 4481 & 14719 & 245 \\
\hline 2008 Aug. 15.19 & 4481 & 13201 & 195 & 2010 Jun. 14.44 & 4481 & $10551 / 3$ & 133 & 2005 Aug. 24.45 & 3933 & 14722 & 275 \\
\hline 2010 Feb. 20.57 & 4481 & $3001 / 2$ & 160 & 2010 Jul. 31.32 & 4481 & $14088 / 9$ & 170 & 2007 Aug. 25.43 & 3933 & 11757 & 165 \\
\hline 2010 May 17.33 & 3933 & 9879 & 100 & 2010 Aug. 19.29 & 4481 & $15737 / 8$ & 140 & 2008 Aug. 16.48 & 4481 & 13262 & 195 \\
\hline 2010 Jun. 12.33 & 3933 & $10450 / 1$ & 112 & 2010 Aug. 20.32 & 4481 & $15787 / 8$ & 124 & 2009 Oct. 6.33 & 4481 & 25411 & 210 \\
\hline 2010 Jun. 15.35 & 4481 & 10654 & 112 & 2010 Sep. 10.22 & 4481 & $16749 / 0$ & 160 & 2010 Feb. 17.10 & 4481 & 2812 & 200 \\
\hline
\end{tabular}

Notes. ${ }^{(*)}$ Denotes a definite or tentative $\lambda$ Boo classification.

Mg II $\lambda 4481 \AA$ doublet - with sufficient frequency to cover the possibility of spectrum changes caused by binary movement in periods from a few days to a few years. As the project progressed, we set a minimum of 5 observations per wavelength region, spaced (as far as possible) by 1 day, a few days, a few weeks and a few years. The list of our observations (Table 2) is restricted to that minimum, and discards those with $S / N<100$. It includes the UT date, the archive file number and the measured $\mathrm{S} / \mathrm{N}$, the last being derived as an average of three measurements made in the continuum. Co-added spectra are evident from their file numbers. Our insistence on a full set of spectra at both wavelength regions was in fact a redundancy, since in no case did a spectrum at one wavelength region suggest a conclusion that could not be reached by examining the other wavelength region instead. However, it did provide a useful variety of material for synthetic-spectrum fitting and RV measurements.

As explained below, three stars ( $\lambda$ Aql, HR 6878 and HR 7903) exhibited fairly rapid line-profile variations and we consequently observed their spectra on numerous occasions, mostly when the observational material was otherwise complete. The entries listed in Table 2 were selected to match the frequencies that characterised the observations of the other stars.

\section{What the spectra showed}

The spectra showed nothing to suggest that their sources are double-lined binaries.

The evidence on which that conclusion is based is reproduced in Figs. 1 and 2, where the observed spectra of the two 
A\&A 547, A8 (2012)
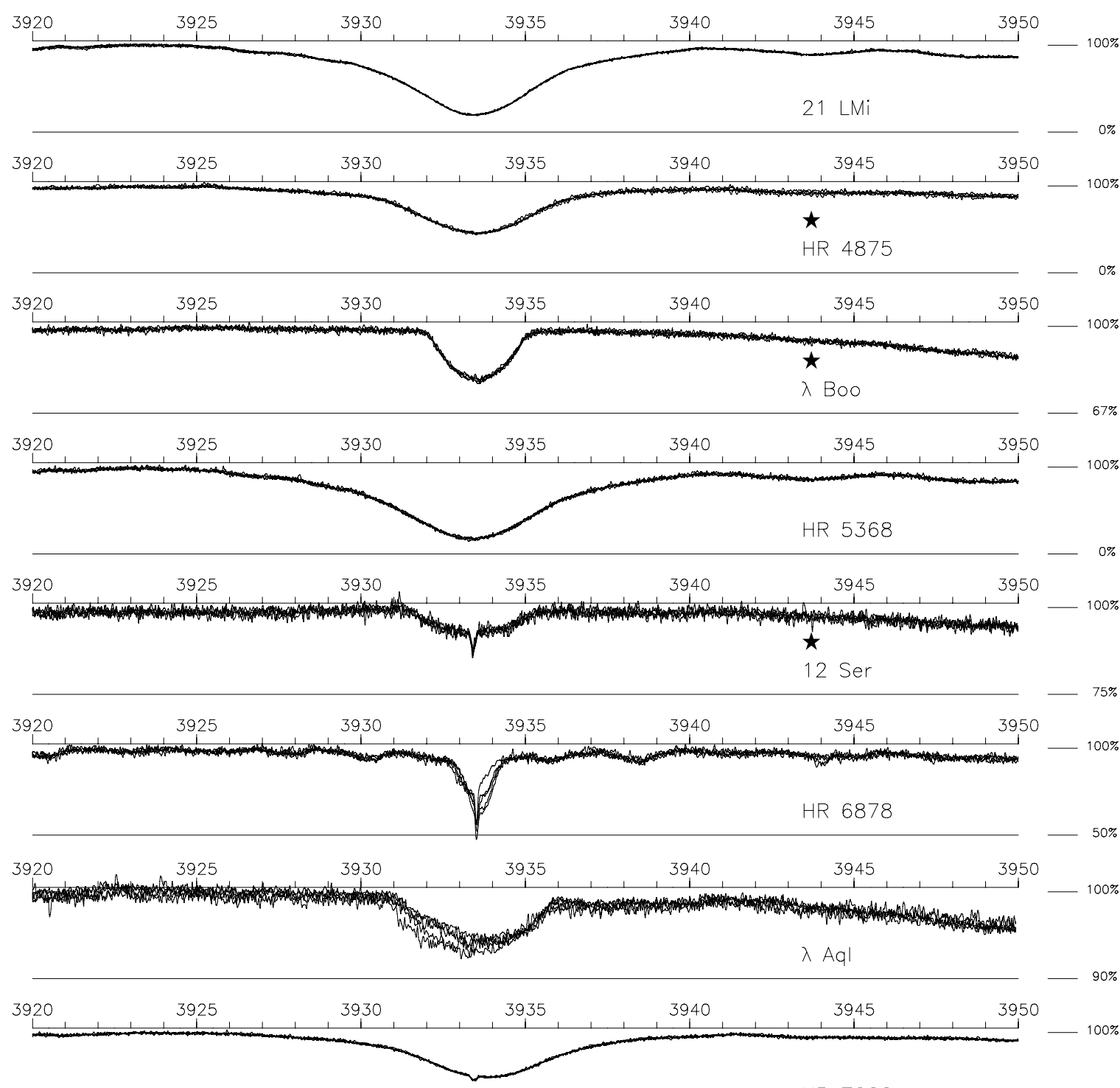

HR 7288
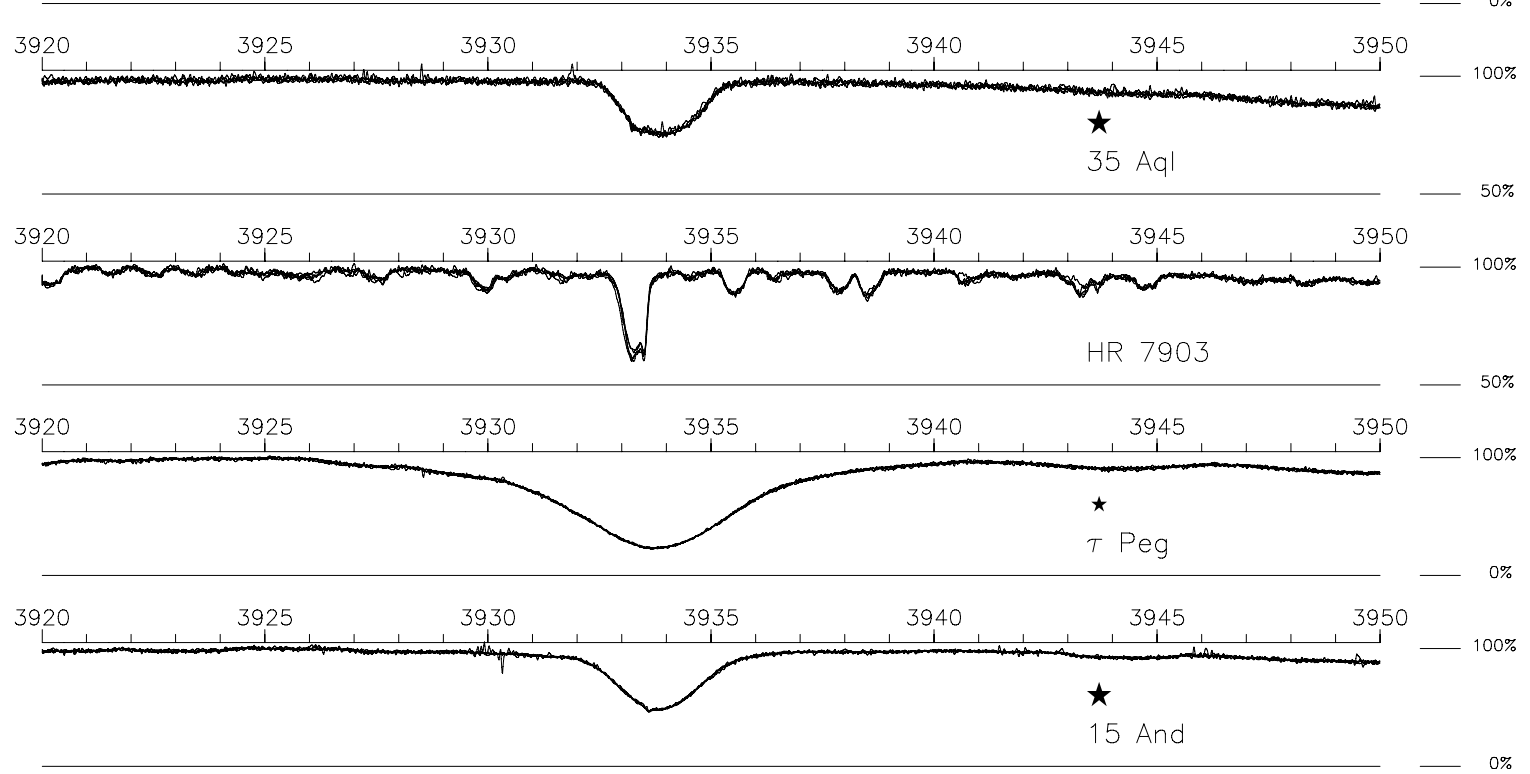

Fig. 1. In each panel we have superimposed the 5 observations of the Ca II K-line region listed in Table 2. The vertical scales have been variously enlarged, as indicated by the labels on the right of each panel. An asterisk indicates that the star has a $\lambda$ Boo classification; a small asterisk denotes that the classification is only probable. 


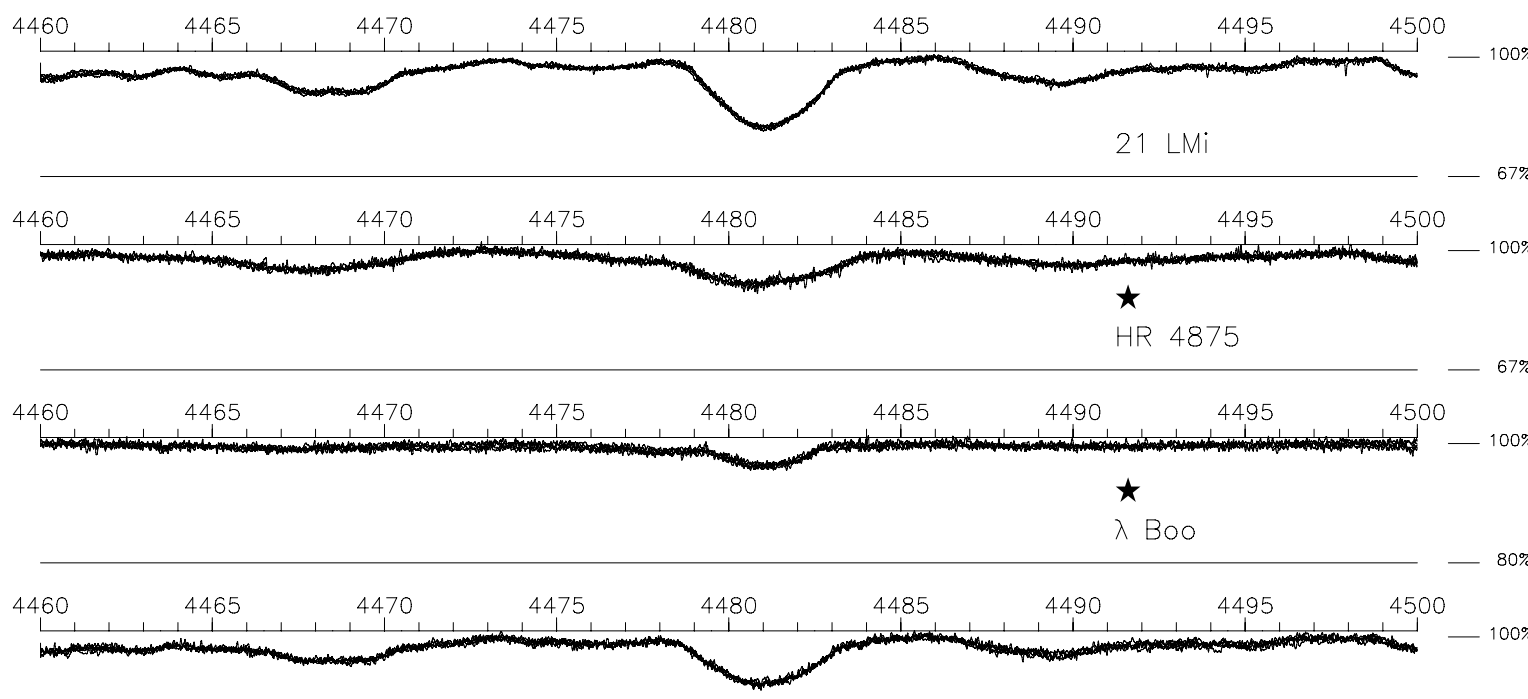

HR 5368

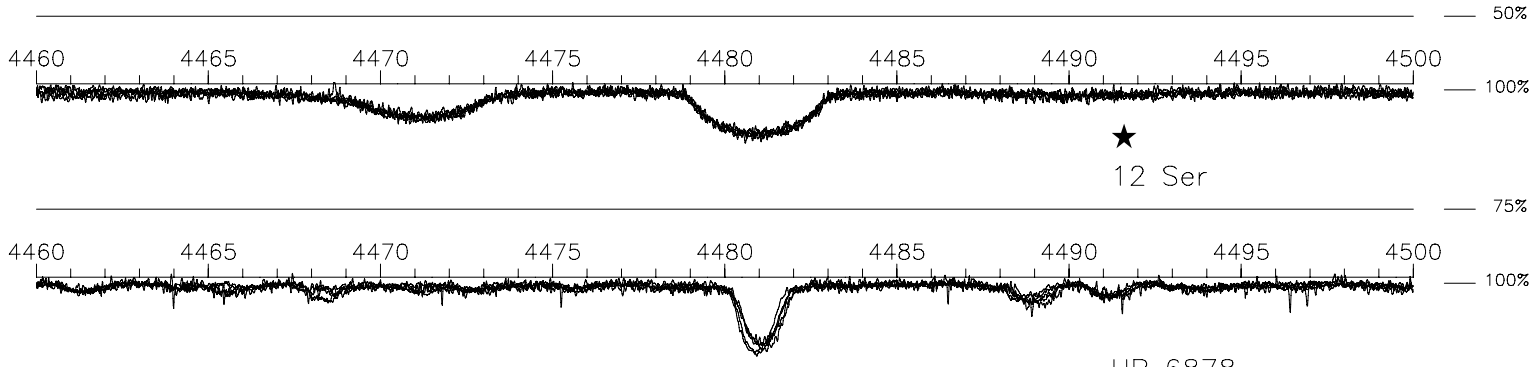

HR 6878

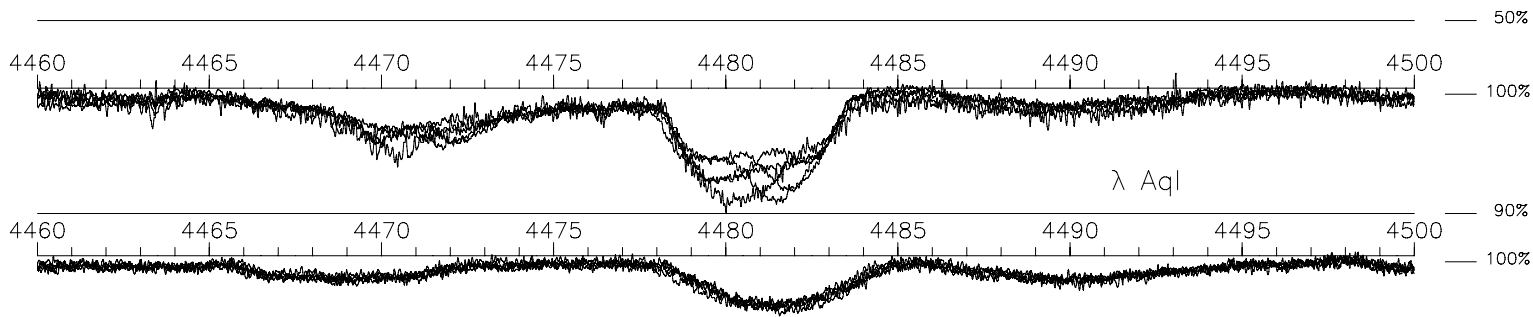

HR 7288

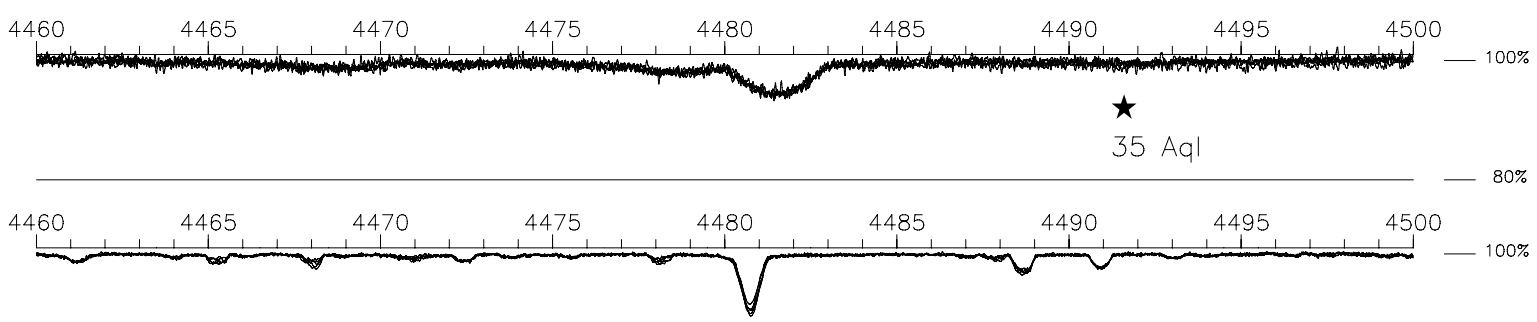

HR 7903
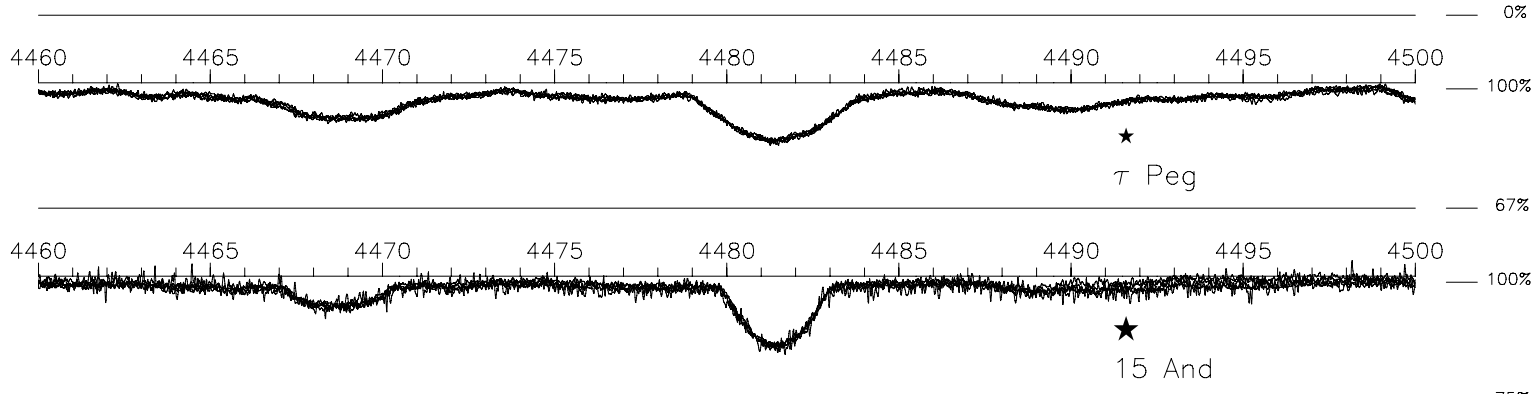

Fig. 2. In each panel we have superimposed the 5 observations of the $\mathrm{Mg}$ II $\lambda 4481$ Å region listed in Table 2. Other features are as given for Fig. 1 . 
wavelength regions for each of the 12 stars listed in Table 2 have been superimposed. Since the spectra were reduced in the heliocentric rest-frame, any variation in velocity that could be attributable to orbital motion would immediately be obvious unless its value were only a few $\mathrm{km} \mathrm{s}^{-1}$, or the orbit were nearly face-on. We comment on individual systems in Sect. 7.

Stars in the $T_{\text {eff }}$ range spanned by our sample are notorious for their high frequency $(\gtrsim 30 \%)$ of spectrum peculiarities, and $25 \%$ of our sample ( $\lambda$ Aql, HR 6878 and HR 7903) indicated spectrum changes which could be so diagnosed - a frequency which reflected the general tendency even if the sample was not completely unbiassed. Figures 1 and 2 illustrate characteristic line-profile changes in the spectra of two of them, and all three will be the subject of a future paper. We summarise our current information about them in Sects. 7.6, 7.7 and 7.10, but we can state here that the variations which their line profiles exhibit resemble those caused by surface chemical inhomogeneities, and cannot be reconciled with the hypothesis that they are composite-spectrum binaries.

None of the stars in our sample, including the three probable CP stars, exhibited the variations in line-width and line-depth which would be expected in a composite spectrum (SB2 system) of two similar, orbiting stars of comparable mass, nor the velocity excursions which would be expected if the component stars were of unequal mass and luminosity (SB1 system). To illustrate that point, we computed composite profiles of the $\mathrm{Mg}$ II doublet at $\lambda 4481 \AA$ for the case of (a) a pair of identical mid-A dwarfs with $T_{\text {eff }}=8000 \mathrm{~K}$ and a projected rotation of $120 \mathrm{~km} \mathrm{~s}^{-1}$ (as characterizing the rotational velocity of the 6 definite or tentative $\lambda$ Boo stars in our sample) and separated by velocities varying from 0 to $100 \mathrm{~km} \mathrm{~s}^{-1}$ (see Fig. 3a), and (b) the same pair but differing in brightness by a factor of 2 (see Fig. 3b). For the sake of clarity, Fig. $3 \mathrm{~b}$ shows the resulting spectra during only onehalf of a complete orbit; in Fig. 3a the two halves are of course identical.

A composite spectrum formed from two somewhat similar stars and rotating at $\sim 120 \mathrm{~km} \mathrm{~s}^{-1}$ would exhibit line-broadening but not complete isolation of the component lines unless the orbital velocity separation were about twice that amount. Such velocity amplitudes imply periods of at most a few days, and in all probability the component stars would be in synchronous rotation. For a mid-A star with a radius of $2.5 R_{\odot}$ and rotation axis tilted at $45^{\circ}$ to the line of sight the rotation period corresponding to such rotation is $\sim 18 \mathrm{~h}$, or longer if the star is larger (as a hotter one may well be). Most of the stars in our sample do have rotational velocities of $120-150 \mathrm{~km} \mathrm{~s}^{-1}$ (see Table 4), but for them also to be in binary systems with comparable semiamplitudes, i.e. with periods of the order of a day, variations in the composite line-profiles would be evident in at least some of the systems - yet 9 of the 12 spectra illustrated in Figs. 1 and 2 show no hint of line-profile blurring. It is extremely improbable that every one of our observations happened upon single-lined phases in them all. Only in the case of the three probable CP stars do we see any variation in line profiles; but they do not resemble those illustrated in Figs. $3 a$ and b, and further monitoring, particularly of the changes in line-depth, confirmed that they could not be due to an SB2 nature.

Faraggiana \& Bonifacio (1999) and F+2004 maintain that the apparent low metallicity of a $\lambda$ Boo star could be fully accounted for by the continuum of one component veiling that of its companion. However, our models did not reproduce a line weakening of the dramatic magnitude which is characteristic of the $\lambda$ Boo class. It could possibly be achieved if one member of the binary were hot enough to have a featureless spectrum, but the colour indices of the system, as well as the appearance of the resulting spectra, would quickly reveal such a companion.

Half of the sample also display narrow absorption features near the core of the $\mathrm{Ca}$ II $K$ line. Whether the sources are interstellar or circumstellar is hard to judge unambiguously (see Sect. 6), though all but one of the six are $<170 \mathrm{pc}$ distant (Table 4).

Two of the stars classified as $\lambda$ Boo (35 Aql and $\lambda$ Boo itself) show weak absorption near $\lambda 4479 \AA$ (see Fig. 4) whose provenance is at present unclear; it is present in 15 And too, though is less well defined because the metal deficiency of 15 And is an order of magnitude less extreme so its spectrum is less uncluttered. HR 4875, another star classified as $\lambda$ Boo, also shows a similar feature but its spectrum is more confused with other lines because the star is $\sim 1000 \mathrm{~K}$ cooler than $35 \mathrm{Aql}$ and $\lambda$ Boo and is also an order of magnitude less weak in metals. However, the feature is not present in $21 \mathrm{LMi}, 12 \mathrm{Ser}$, HR 7288 or HR 7903, and almost certainly not in HR 5368, HR 6878, $\lambda$ Aql or $\tau$ Peg. There is no obvious identification for it in published lists of atomic-line data, so the fact that it appears so indisputably in stars whose metallicities are only 1 or $2 \%$ of that of the Sun is the more remarkable. There is no comparable feature on the short-wavelength side of the Ca II K line in those stars, so it does not seem likely that the feature at $\lambda 4479 \AA$ can be a signature of a binary companion.

\section{Radial velocity measurements}

RVs of the sample stars were measured on all the spectra listed in Table 2, and also on a few others with $S / N<100$, by crosscorrelation with appropriate synthetic spectra. We treated all the spectra similarly: the RV was first measured from all the features in each of the two observed regions ("UV" and "blue"), and then from the $K$ line and the Mg II doublet alone. Table 3 includes those four measurements for each star; the uncertainties quoted are the calculated standard deviations. The means $\left(V_{\mathrm{m}}\right)$ in Col. 5 were derived by averaging Cols. 2 and 3, except as stated. In fact, only the three coolest stars in the sample (21 LMi, HR 4875 and $\tau$ Peg) have measureable spectral features besides Balmer lines, the Ca II $K$ line and the Mg II $\lambda 4481 \AA$ line; in $\lambda$ Boo the $\mathrm{Mg}$ II line barely reaches a central depth of $4 \%$ so $V_{\mathrm{m}}$ for that star omits the blue region.

Our cross-correlation of the UV region required truncating the spectra at 90 or $95 \%$ of the continuum height in order to accommodate the absence of the extreme violet wing of $\mathrm{H} 8$ from our observations, and we thereby unavoidably reduced or excluded the contribution from the $\mathrm{K}$ line if it were less than 5 or $10 \%$ deep, as is the case in the metal-poor stars $\lambda$ Boo, 12 Ser and $35 \mathrm{Aql}$.

We derived RV measurements for $\lambda \mathrm{Aql}$ as for the other 11 stars for the sake of completeness, though it is clear from Figs. 1 and 2 that the measurements will only be some rather meaningless average. The comments in the rest of this section do not apply to that star.

The accuracy of RV measurements in stars such as these depends critically upon the quality and nature of the observations. For pre-digital measurers the challenges of setting a cross-hair visually at the centroid of a naturally wide Balmer line that is further broadened by a rotation of the order of $150-200 \mathrm{~km} \mathrm{~s}^{-1}$ should not be underestimated, especially when only a few such lines are available for measurement. Though intrinsically narrower in these stars, the Ca II K-line may harbour a sharp IS or CS contribution which, if sufficiently strong 


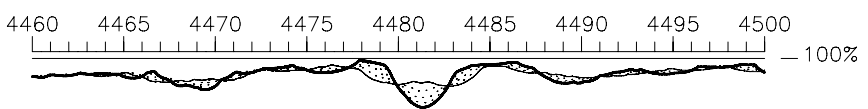

(a)

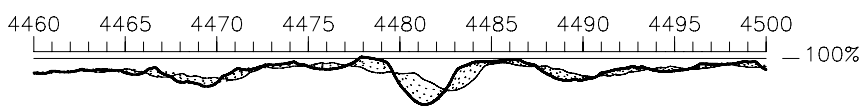

(b)

Fig. 3. a) Synthetic spectra of two identical dwarfs with $T_{\text {eff }}=8000 \mathrm{~K}$, and mutually displaced by projected velocities of $0,40,80,120,160$ and $200 \mathrm{~km} \mathrm{~s}^{-1}$ to mimic a composite-spectrum binary (SB2). The continuous thick line represents the single-lined state, and the normal continuous line the maximum velocity displacement; the intermediate cases are drawn dotted. b) Similar to a), but the two otherwise identical stellar spectra differ in brightness by a factor of 2 . For clarity our computed spectra represent only one-half of the orbit. In neither case do the composite spectra suggest a single metal-deficient object.

Table 3. RV measurements at different wavelengths.

\begin{tabular}{|c|c|c|c|c|c|c|c|c|}
\hline Star & HD & UV & Ca II K & Blue & $\lambda 4481 \AA$ & $V_{\mathrm{m}}$ & $B S C$ & Comments \\
\hline & & (1) & (2) & (3) & (4) & (5) & (6) & \\
\hline $21 \mathrm{LMi}$ & 87696 & $-14.5 \pm 0.9$ & $-17.7 \pm 0.8$ & $-17.0 \pm 0.4$ & $-21.3 \pm 1.1$ & $-17.4 \pm 0.7$ & $-18 \mathrm{~V}$ & \\
\hline HR $4875^{*}$ & 111604 & $-10.8 \pm 1.1$ & $-10.5 \pm 1.2$ & $-10.5 \pm 1.0^{\dagger}$ & $-32.4 \pm 1.5$ & $-10.5 \pm 1.0$ & $-14 \mathrm{~V}$ & $V_{\mathrm{m}}$ omits $\lambda 4481 \AA$ \\
\hline$\lambda$ Boo* $^{*}$ & 125162 & $-9.7 \pm 3.7$ & $-9.8 \pm 0.8$ & $-17.4 \pm 2.6$ & $-17.6 \pm 1.5$ & $-9.8 \pm 0.8$ & -8 & $V_{\mathrm{m}}$ from $(1)+(2)$ \\
\hline HR 5368 & 125489 & $-18.4 \pm 1.3$ & $-21.5 \pm 0.3$ & $-20.6 \pm 1.0$ & $-25.4 \pm 0.4$ & $-21.0 \pm 0.9$ & $-22 \mathrm{~V}$ & \\
\hline 12 Ser$^{*}$ & 138527 & $-15.7 \pm 2.3$ & $-18.1 \pm 1.9$ & $-18.6 \pm 0.9$ & $-17.8 \pm 1.0$ & $-18.3 \pm 1.5$ & $-18 \mathrm{~V}$ & \\
\hline HR 6878 & 169009 & $-10.4 \pm 4.9$ & $-10.7 \pm 6.4$ & $-13.2 \pm 4.6$ & $-12.7 \pm 5.2$ & $-12.0 \pm 5.7^{\S}$ & $-24 \mathrm{~V}$ & $\mathrm{CP}$ \\
\hline$\lambda \mathrm{Aql}$ & 177756 & $-21.5 \pm 9.2$ & $-15.4 \pm 12.8$ & $-26.3 \pm 6.2$ & $-27.3 \pm 5.9$ & $-22.3 \pm 8.9^{\S}$ & $-12 \mathrm{~V}$ & $V_{\mathrm{m}}$ from $(1)+(3) . \mathrm{CP}$ \\
\hline HR 7288 & 179791 & $+20.4 \pm 1.0$ & $+13.8 \pm 1.1$ & $+11.3 \pm 1.3$ & $+13.3 \pm 2.6$ & $+12.7 \pm 1.7$ & +9 V? & \\
\hline $35 \mathrm{Aql}^{*}$ & 183324 & $+18.5 \pm 2.3$ & $+16.0 \pm 0.5$ & $+16.8 \pm 1.6$ & $+16.7 \pm 1.7$ & $+16.3 \pm 1.2$ & $+12 \mathrm{~V}$ & \\
\hline HR 7903 & 196821 & $-29.1 \pm 1.3$ & $-28.2 \pm 1.0$ & $-32.7 \pm 1.1$ & $-32.7 \pm 1.1$ & $-30.4 \pm 2.4^{\S}$ & $-37 \mathrm{SB} ?$ & $\mathrm{CP}$ \\
\hline$\tau$ Peg* & 220061 & $+7.1 \pm 0.9$ & $+4.4 \pm 0.5$ & $+8.2 \pm 1.0$ & $+4.9 \pm 2.2$ & $+6.1 \pm 2.0$ & $+16 \mathrm{~V}$ & \\
\hline 15 And* & 221756 & $+15.2 \pm 1.0$ & $+13.5 \pm 0.6$ & $+12.4 \pm 0.8$ & $+12.7 \pm 0.9$ & $+12.9 \pm 0.9$ & $+13 \mathrm{~V}$ & \\
\hline
\end{tabular}

Notes. All values are in $\mathrm{km} \mathrm{s}^{-1} .{ }^{(*)}$ Denotes a definite or tentative $\lambda$ Boo classification. ${ }^{(\dagger)} \mathrm{RV}=-20.8 \pm 2.1 \mathrm{~km} \mathrm{~s}^{-1}$ if the Mg II blend is included. (§) The variable line profiles in a CP star can cause a large s.d. in measured RVs.

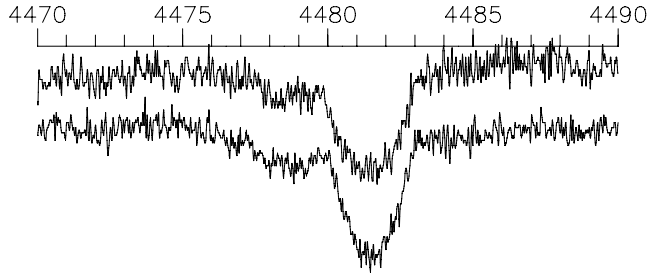

Fig. 4. The $\mathrm{Mg}$ II region in two confirmed $\lambda$ Boo stars: $\lambda$ Boo (upper) and 35 Aql (lower), illustrating the unidentified absorption near $\lambda 4479 \AA$. There is no corresponding absorption shortward of the $\mathrm{Ca}$ II $\mathrm{K}$ lines in those stars, so the cause is unlikely to be binarity.

and displaced relative to the star, can distort the measurement of RV. Furthermore, any swerves in the observed stellar continuum across the broad wings of the Balmer lines are not easily discernible, and will also bias the RV(UV) measurements which depend on those features. Table 3 shows several cases of disharmony in which the precision of the RVs from the Balmer lines on the one hand and from the $\mathrm{Ca}$ II $\mathrm{K}$ line alone, on the other, is better than their inter-agreement (apparent accuracy). Given all the caveats mentioned above, we chose to derive $V_{\mathrm{m}}(\mathrm{Col} .5)$ by averaging those from Col. 2 (the $\mathrm{Ca}$ II $\mathrm{K}$ line alone) and Col. 3 (the blue region).

The inter-agreement between the UV and blue regions is acceptable except for HR 4875, $\lambda$ Boo and HR 7288, where the measurements show a scatter that substantially exceeds the respective precisions and suggest one or more sources of bias, though rather surprisingly there is no obvious "drag" that could be attributed to the presence of a K-line "pip". In the cooler stars, blending compounded by substantial rotational blurring is a very probable cause of $\mathrm{RV}$ errors in the $\mathrm{Mg}$ II region. The blue spectra of HR 4875 and $\lambda$ Boo, and also that of $35 \mathrm{Aql}$, show a broad absorption at $\lambda 4479 \AA$ that is not matched in the synthetic spectra. The spectrum of HR 4875 is rich enough in lines that we could exclude the $\lambda$ 4479-4481 $\AA$ blend and determine the RV from other features in that region; the value of $-10.5 \pm 1.0 \mathrm{~km} \mathrm{~s}^{-1}$ which we then derived was markedly different from the $-20.8 \mathrm{~km} \mathrm{~s}^{-1}$ which resulted when the blend was included. Its $\lambda 4479 \AA$ component may therefore have constituted a serious bias for that star. However, $35 \mathrm{Aql}$ and $\lambda$ Boo are not only $\sim 1000 \mathrm{~K}$ hotter but also substantially more metalpoor and have lower projected rotational velocities, resulting in weaker $\mathrm{Mg}$ II lines that are not significantly blended with the $\lambda 4479 \AA$ feature. In fact, the lines in the blue region of those stars are all so weak that when the $\lambda 4479-4481 \AA$ blend was excluded there was little else to measure, and the cross-correlation was dominated by noise.

Column 6 of Table 3 lists the RVs for our stars as given in the BSC (Hoffleit 1982); they were taken from either the General Catalog of Stellar Radial Velocities (Wilson 1953) or its Revision (Evans 1967). Comparison between Cols. 5 and 6 is instructive. The label " $V$ " or " $V$ ?" was appended by the $B S C$ "merely to indicate that the array of published values appears to have a greater spread than the average probable errors would warrant", though Table 3 illustrates quite clearly how a measurement of RV in B or A-type stars can depend on the spectral region as well as on the technique adopted and the quality of the observation. The upshot is that the literature has probably mis-labelled as "RV variable" a large number of warm/hot stars which are in fact single. Those self-same mis-labels 
Table 4. Physical parameters for the sample stars.

\begin{tabular}{|c|c|c|c|c|c|c|c|c|c|}
\hline 10 Star & HD & $\begin{array}{l}T_{\text {eff }} \\
(\mathrm{K})\end{array}$ & $\log g$ & $E_{B-V}$ & {$[\mathrm{M} / \mathrm{H}]$} & $\begin{array}{c}v \sin i \\
\left(\mathrm{~km} \mathrm{~s}^{-1}\right)\end{array}$ & $\begin{array}{c}d^{\dagger} \\
(\mathrm{pc})\end{array}$ & $\begin{array}{l}\text { K-line } \\
\text { "pip" }\end{array}$ & Comments \\
\hline $21 \mathrm{LMi}$ & 87696 & 7850 & 3.88 & 0.00 & +0.15 & 130 & 28 & $\mathrm{~N}$ & {$[\mathrm{Ca} / \mathrm{Fe}]-0.1,[\mathrm{Mg} / \mathrm{Fe}]+0.1$} \\
\hline HR $4875^{*}$ & 111604 & 7790 & 3.62 & 0.00 & -0.75 & 170 & 110 & $\mathrm{~N}$ & {$[\mathrm{Ca} / \mathrm{Fe}]+0.1,[\mathrm{Mg} / \mathrm{Fe}]+0.3$} \\
\hline$\lambda$ Boo* $^{*}$ & 125162 & 8570 & 3.95 & 0.00 & -2.00 & 110 & 30 & $\mathrm{~N}$ ? & {$[\mathrm{Ca} / \mathrm{Fe}]+0.3$} \\
\hline HR 5368 & 125489 & 7820 & 3.88 & 0.005 & +0.2 & 130 & 65 & $\mathrm{~N}$ & \\
\hline 12 Ser* $^{*}$ & 138527 & 11480 & 4.09 & 0.045 & -0.80 & 135 & 145 & $\mathrm{Y}$ & {$[\mathrm{Mg} / \mathrm{Fe}]+0.2$} \\
\hline HR 6878 & 169009 & 10960 & 4.31 & 0.25 & $0.00: \mathrm{V}$ & 50 & 100 & Y & {$[\mathrm{Ca} / \mathrm{Fe}]-0.3,[\mathrm{Mg} / \mathrm{Fe}]-0.2 \mathrm{CP}$} \\
\hline$\lambda \mathrm{Aql}$ & 177756 & 11680 & 4.06 & 0.00 & $-0.50: \mathrm{V}$ & 170 & 60 & $\mathrm{~N}$ & $\mathrm{CP}$ \\
\hline HR 7288 & 179791 & 8860 & 3.42 & 0.05 & +0.30 & 200 & 150 & Y & {$[\mathrm{Mg} / \mathrm{Fe}]+0.2$} \\
\hline $35 \mathrm{Aql}^{*}$ & 183324 & 8900 & 4.28 & 0.03 & -1.80 & 95 & 160 & $\mathrm{Y}$ & {$[\mathrm{Ca} / \mathrm{Fe}]+0.4$} \\
\hline HR 7903 & 196821 & 10000 & 3.47 & 0.00 & $0.00: \mathrm{V}$ & 20 & 220 & Y & {$[\mathrm{Ca} / \mathrm{Fe}]-1.0,[\mathrm{Mg} / \mathrm{Fe}]-0.6 \mathrm{CP}$} \\
\hline$\tau$ Peg $^{*}$ & 220061 & 7740 & 3.63 & 0.00 & -0.30 & 140 & 50 & $\mathrm{~N}$ & \\
\hline 15 And* & 221756 & 8520 & 3.78 & 0.00 & -0.75 & 100 & 80 & Y & \\
\hline
\end{tabular}

Notes. ${ }^{(*)}$ Denotes a definite or tentative $\lambda$ Boo classification. ${ }^{(\dagger)}$ Derived from the HIPPARcos parallax (van Leeuwen 2007). Uncertainties are estimated as $\pm 100 \mathrm{~K}$ for $T_{\text {eff }}$, and \pm 0.10 for $\log g$ and $[\mathrm{M} / \mathrm{H}]$.

encouraged $\mathrm{G}+2003$ and collaborators to suppose that stars designated as $\lambda$ Boo candidates are actually SB systems.

It is relatively easy to state in a publication that recorded values show enough discordance to signal a physical cause, but it takes a great deal of time, effort and observational resources to prove that a property thus labelled "variable" is in fact constant. In the present instance, all 12 stars except $\lambda$ Aql have RVs that are constant to within a small margin of uncertainty, mostly no more than $\sim 3 \mathrm{~km} \mathrm{~s}^{-1}$, yet all but one (and interestingly it is $\lambda$ Boo itself which is the exception) are labelled " $V$ " or " $V$ ?" in the $B S C$, a popular catalogue upon which many observers and theoreticians rely.

\section{Physical parameters of the sample stars}

Classification-dispersion spectra of the 12 stars in the sample were obtained at Appalachian State University's Dark Sky Observatory (DSO). Those spectra have a resolution of $1.8 \AA / 2$ pixels and cover the range $\lambda \lambda 3800-4620 \AA$; they were recorded on a thinned, back-illuminated $1024 \times 1024$ Tektronix $\mathrm{CCD}$ with the $0.8-\mathrm{m}$ telescope and $\mathrm{G} / \mathrm{M}$ spectrograph fitted with a 1200 -line $\mathrm{mm}^{-1}$ grating. An Fe-Ar hollow-cathode lamp was observed for wavelength calibrations, and the spectra were reduced with standard IRAF ${ }^{1}$ routines.

Good approximations to each star's physical parameters ( $T_{\text {eff }}, \log g$ and overall metallicity $[\mathrm{M} / \mathrm{H}]$ ) were derived by the SIMPLEX method (Gray et al. 2001, 2003). The SIMPLEX method derives the physical parameters by minimizing a $\chi^{2}$ statistic formed from the point-to-point squared differences between the synthetic and observed classification-resolution spectra plus a similar sum over the squared differences between the observed and synthetic fluxes. The observed fluxes (stellar energy distribution) were based on Strömgren photometry and, when available, included UV data from either the TD1 (Thompson et al. 1978) or the IUE satellites. For the present paper, the theoretical fluxes and synthetic spectra were calculated from ATLAS9 models (Castelli \& Kurucz 2003) and the spectral synthesis code SPECTRUM (Gray \& Corbally 1994).

\footnotetext{
1 IRAF is distributed by the National Optical Astronomy Observatory, which is operated by the Association of Universities for Research in Astronomy, Inc., under cooperative agreement with the USA National Science Foundation.
}

The provisional stellar parameters thus derived then became the bases of new ATLAS9 models, from which higher-resolution synthetic spectra were calculated using SPECTRUM ${ }^{2}$ and compared to the observed DAO spectra. Modest iterations were introduced as necessary. A microturbulence of $2.0 \mathrm{~km} \mathrm{~s}^{-1}$ was assumed for all models. Each stellar rotational velocity, $v_{\text {rot }}$, was derived by optimizing the fits to the DAO spectra. The parameters for the 12 sample stars are listed in Table 4. Reddening values, $E_{B-V}$, were derived from tabulated Strömgren colour indices but slightly adjusted if necessary to optimize the SIMPLEX fits. The positions of the 12 stars in the HR diagram are shown in Fig. 5. Overall uncertainties were estimated as $\pm 100 \mathrm{~K}$ for $T_{\text {eff }}$ and \pm 0.10 dex for $\log g,[\mathrm{M} / \mathrm{H}]$; even where there was a little difficulty in fitting all the photometric data for a star unambiguously (as occurred for $\lambda$ Boo), the comparisons with the highresolution spectra served to tighten the precisions to the values just quoted.

The 6 stars that have definite or tentative $\lambda$ Boo classifications are all metal-poor to some degree - nearly 2 dex in $\lambda$ Boo and $35 \mathrm{Aql}$, and a factor of about 6 in three others. Only $\tau$ Peg (whose classification as a $\lambda$ Boo star is questionable) has a metal deficiency that is not particularly extreme (a factor of 2). The 3 apparently normal stars have solar abundances, or are slightly metal-strong; the $3 \mathrm{CP}$ stars have abnormal abundance ratios and probably cannot be treated rigorously by the analysis methods used here. Figure 6 compares synthetic to observed spectra for the 6 objects which have definite or tentative $\lambda$ Boo classifications.

\section{Sharp features in the $\mathrm{Ca}$ II $\mathrm{K}$ line}

A sharp absorption feature ("pip") appears in the Ca II K line of half of the sample (see Fig. 1). The pip is unmistakeable in 12 Ser, HR 6878, HR 7288 and HR 7903; in 15 And it is weak but persistent, while in the K-line profile of $35 \mathrm{Aql}$ it adds a "squareness" on the short-wavelength side that is present in all of our spectra of that star. It is quite prominent in the most distant (HR 7903, at 220 pc) but is strongest in HR 6878, which is only half that distance. Its presence in $\lambda$ Boo is hard to confirm; the hint of such a feature in the centre of its $\mathrm{K}$ line in

2 http://wwW.physics.appstate.edu/spectrum/spectrum.html 


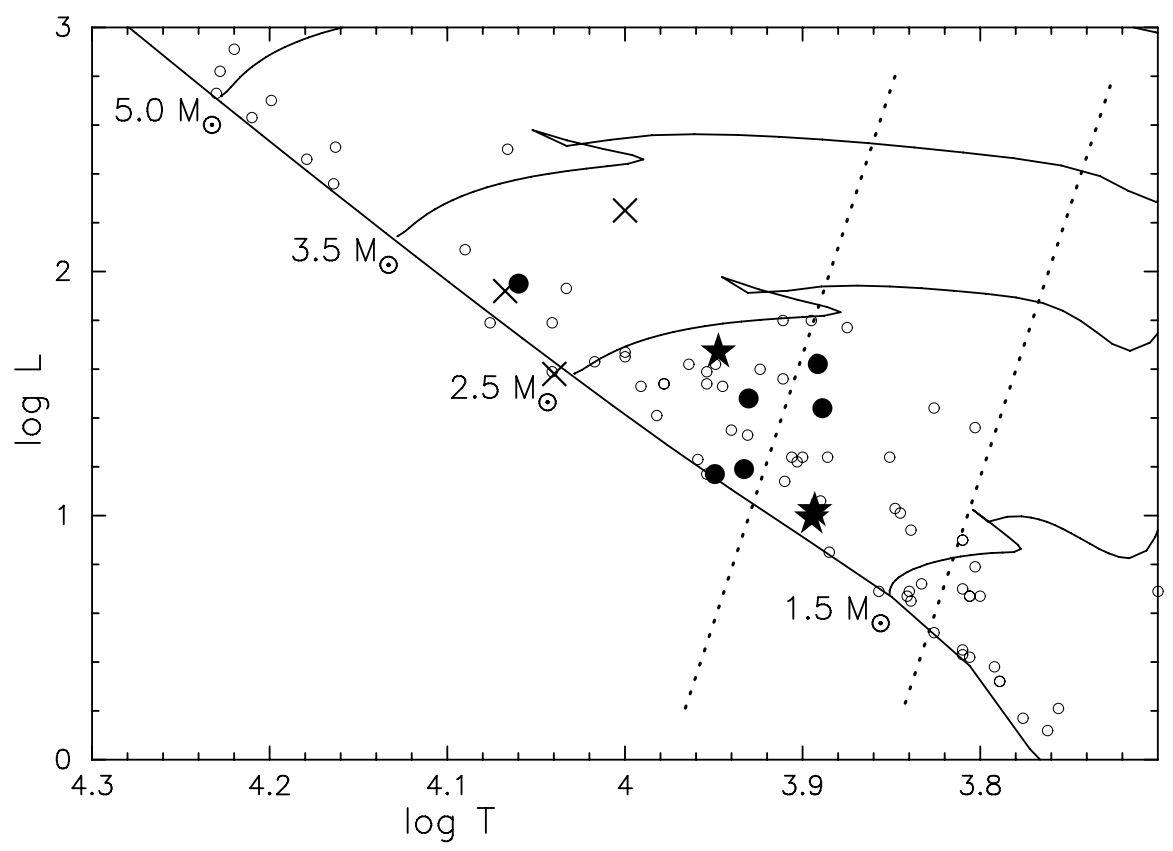

Fig. 5. The positions of the 12 stars in the $\log L, \log T$ plane. The smooth line and the small open circles dots, delineating the main sequence, are from Andersen (1991). The evolutionary tracks, drawn for specific masses as indicated, are described by Pols et al. (1998) and were calculated from software now in the public domain. The six large dots represent the definite or suspected $\lambda$ Boo stars, the crosses the three CP stars, and the asterisks the "normal" members of the sample. The dotted lines suggest the limits of the instability strip.

Table 5. Narrow components in the Ca II K-line.

\begin{tabular}{lrrrr|rrr}
\hline \hline Star & HD & $\begin{array}{r}d \\
\mathrm{pc})\end{array}$ & $\begin{array}{c}\text { RV of star } \\
\left(\mathrm{km} \mathrm{s}^{-1}\right)^{a}\end{array}$ & $\begin{array}{r}v_{\text {rot }} \\
\left(\mathrm{km} \mathrm{s}^{-1}\right)\end{array}$ & \multicolumn{3}{|c}{ RV of "pip" $\left(\mathrm{km} \mathrm{s}^{-1}\right)$} \\
observed $^{a}$ & rel. to star & rel. to LSR \\
\hline 15 And & 221756 & 80 & +12.9 & 100 & -2.7 & -15.6 & +4.9 \\
HR 6878 & 169009 & 100 & -12.0 & 50 & -9.9 & +2.1 & +2.1 \\
12 Ser* & 138527 & 145 & -18.3 & 135 & -17.5 & +0.8 & -4.4 \\
HR 7288 & 179791 & 150 & +12.7 & 200 & -15.2 & -27.9 & -1.0 \\
35 Aql* & 183324 & 160 & +16.3 & 100 & -31.7 & -48.0 & -18.4 \\
HR 7903 $^{1}$ & 196821 & 220 & -30.4 & 20 & -11.0 & +19.4 & +2.7 \\
\hline
\end{tabular}

Notes. ${ }^{(*)}$ Denotes a definite or tentative $\lambda$ Boo classification. ${ }^{(a)}$ Heliocentric velocity. ${ }^{(b)}$ Local Standard of Rest.

Fig. 1 is probably a noise spike in one of the spectra, and is not convincingly reproduced in all of the other spectra of that star.

It seems unlikely a priori that all the pips seen in this sample share a single common origin. That a pip is not apparent in any star with $d<75$ pc might suggest that it is interstellar (IS) in origin; its non-detection in HR 4875, whose distance is $\sim 110 \mathrm{pc}$, may be due to masking by the strong photospheric $K$ line in a star that cool. However, since some hypotheses about the $\lambda$ Boo stars propose surface contamination by accreted circumstellar (CS) material, we should examine these K-line pips in case they can offer any information that bears on a CS environment in $\lambda$ Boo stars. Three of the stars in Table 5 have definite or tentative $\lambda$ Boo classifications, so should there be an explanation for those three in terms of CS absorption we need to understand why it is not seen in the other three stars also classified as $\lambda$ Boo, and equally why it appears in stars that do not have $\lambda$ Boo-like spectra.

Eight of the 12 stars in our sample lie within, or close to, the pulsational instability strip; five of those are the confirmed or suspected $\lambda$ Boo stars; only 12 Ser, the hottest in our sample and whose classification as $\lambda$ Boo is only tentative, lies well outside that region. A significant number of all confirmed $\lambda$ Boo stars do in fact exhibit non-radial pulsations (Bohlender et al. 1999), and those can apparently give rise to narrow CS features. Shell and IS features are of course not mutually exclusive, though there is no evidence of multiple structure in any pip.

Our heliocentric RV measurements of the pips are recorded in Table 5, where the stars are arranged in order of increasing distance $d(\mathrm{pc})$ as deduced from their HIPPARcos parallaxes (van Leeuwen 2007). The Table also includes the RV of the star itself (from Table 3) for comparison. The pips appear to have similar widths of $\sim 0.04 \AA$, or $3 \mathrm{~km} \mathrm{~s}^{-1}$, but since that is close to the resolution of the observations their actual widths may be narrower than that.

According to Holweger \& Rentzsch-Holm (1995) and Holweger et al. (1999), the detectability of a CS pip is governed by stellar rotation. They point out that CS features seem to be more readily visible in stars with appreciable projected rotation ( $v \sin i>80 \mathrm{~km} \mathrm{~s}^{-1}$ ), and surmise that if the CS disk responsible for each pip is narrow enough it is only when viewed nearly edge-on that it becomes optically dense enough along the line of sight to cause sufficient absorption. CS features would therefore tend to be detected in (though not necessarily restricted to) stars with high apparent rotation (their sample suggested $v \sin i \gtrsim$ $120 \mathrm{~km} \mathrm{~s}^{-1}$ ). Since both HR 6878 and HR 7903 do display a 

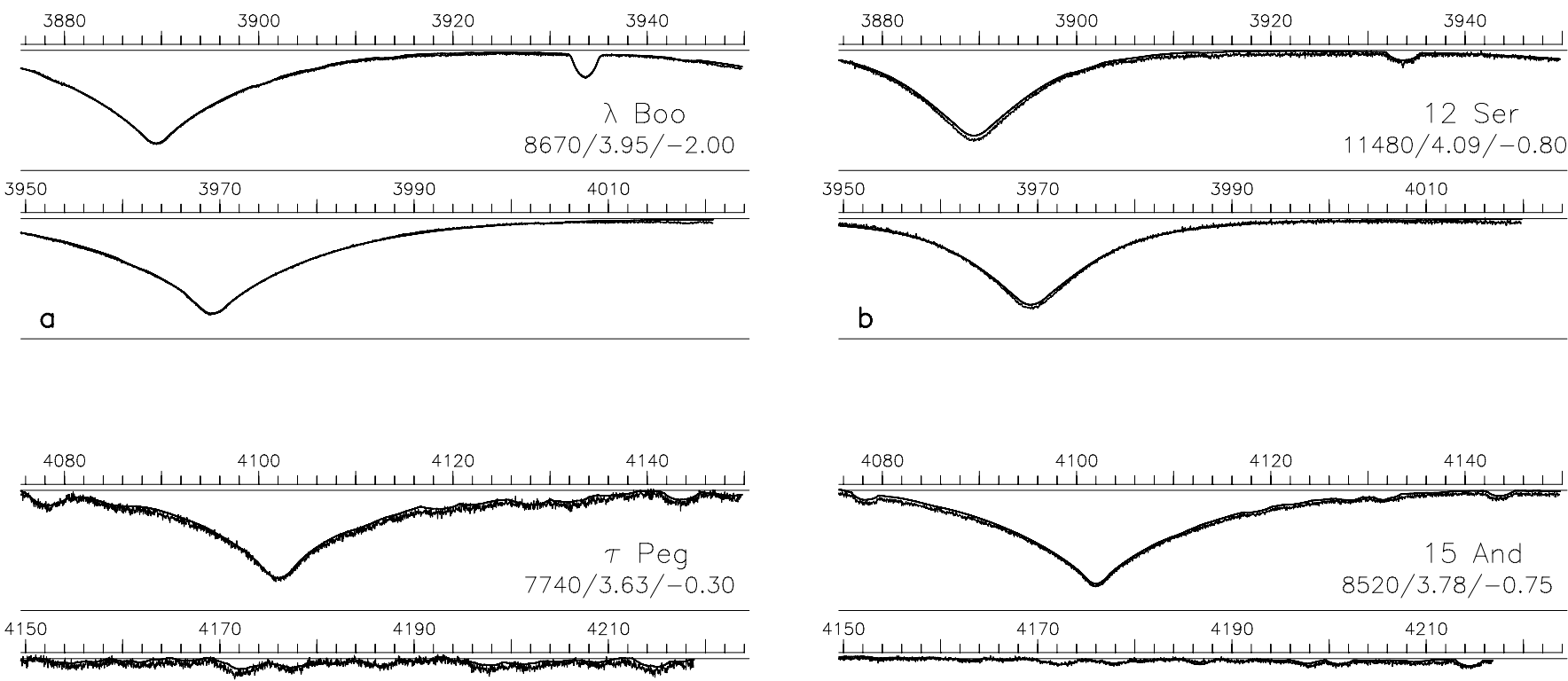

C

d
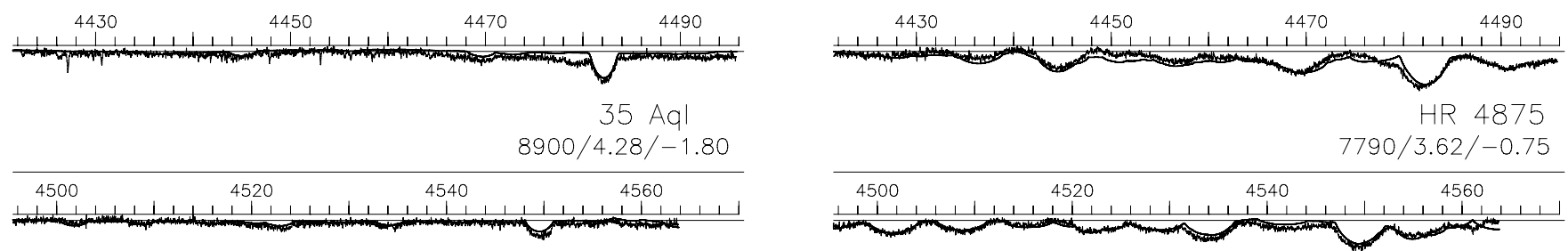

e

$f$

Fig. 6. Spectra of the six definite or probable $\lambda$ Boo stars in our sample are compared with synthetic spectra generated from ATLAS9 models having the parameters listed in Table 4. Panels a) and b) show the Ca II K-line regions of $\lambda$ Boo and 12 Ser, panels c) and d) show the H- $\delta$ regions of $\tau$ Peg and 15 And, while panels e) and f) show the blue regions of 35 Aql and HR 4875.

K-line pip and neither (particularly HR 7903) has high apparent rotation, the pips which are obvious in those spectra would seem to suggest an IS origin for those stars. We note parenthetically that, in their chemical abundance analysis of late-B stars that included HR 6878, Hempel \& Holweger (2003) reported the occurrence of narrow absorption features in the Ca II $\mathrm{K}$ line in some of their sample but rather surprisingly did not mention its appearance in HR 6878, yet in the present study it is the strongest example of all. There is no indication that the pip in that star varies in strength; the variations which we detected in its K-line were caused by variations in the photospheric component, not the pip.

The comprehensive investigation of non-radial pulsations in confirmed $\lambda$ Boo stars by Bohlender et al. (1999) was based on observations of the Na D lines. It included HR 4875, $\lambda$ Boo, $35 \mathrm{Aql}$ and $15 \mathrm{And}$; all four were found to undergo non-radial pulsations, and although $35 \mathrm{Aql}$ and 15 And show only vestigial pips at the $\mathrm{K}$ line they are at blue-shifts similar to those of the corresponding D-line ones reported by Bohlender et al. While it is not suggested that non-radial pulsations are confined to $\lambda$ Boo stars, the Bohlender et al. hypothesis would, if extrapolated, predict CS-like features in all $\lambda$ Boo stars, but produced internally by pulsations rather than externally by a disk.
The fact that three $\lambda$ Boo stars (HR 4875, $\tau$ Peg and $\lambda$ Boo itself) do not show convincing evidence of a K-line pip even though all have adequately high projected rotational velocities to meet the criterion of Holweger et al. (1999), suggests that the narrow-disk hypothesis for $\lambda$ Boo stars does not offer a sufficient observational criterion for classifying $\lambda$ Boo stars. It should be pointed out, however, that HR 4875 and $\tau$ Peg are cool enough that their substantial photospheric $\mathrm{K}$ lines possibly mask any small CS pip. Our observations of the $\mathrm{K}$ lines in this sample are therefore not an adequate basis as evidence for CS material.

An infrared excess thought to be caused by dust from circumstellar debris disks has been recorded by Su et al. (2006) for $\lambda$ Boo, $35 \mathrm{Aql}$ and $15 \mathrm{And}$. Su et al. list the ages of the stars they investigated (giving 313, 10 and $130 \mathrm{Myr}$ for $\lambda$ Boo, $35 \mathrm{Aql}$ and 15 And, respectively), and found a general decrease in excess IR emission with age. However, absorption in the $\mathrm{Ca}$ II $\mathrm{K}$ line is a signature of gas rather than dust, so one may not connect the presence of a K-line pip in $35 \mathrm{Aql}$ and 15 And with the observed IR excess in those stars.

The pips recorded in Table 5 could be interstellar. To that end, in Col. 8 we have corrected the RVs to the Local Standard of Rest (LSR), using rectilinear solar-motion coordinates determined by Francis \& Anderson (2009). IS Na I has been reported 
in HR 6878, 12 Ser, HR 7288 and HR 7903 by various authors at velocities, relative to the Local Standard of Rest (LSR), that agree well enough with our own measurements in Table 5 to suggest that the K-line features in those stars are IS rather than CS. Details of individual cases are discussed in Sect. 7.

Our RV measurements of the K-line pip also have a bearing on the question of binarity. Were the star an SB1 in orbit around a companion, then if the pip were IS we would expect to see displacements as the RV of the star changed relative to the systemic velocity. In all cases observed here, the pip has a constant velocity displacement from the centre of the photospheric line, adding weight to the overall conclusion of this study, namely that we see no evidence that stars classified as $\lambda$ Boo are actually binaries.

\section{Notes on individual stars}

\section{1. $21 L M i=H D 87696$}

$21 \mathrm{LMi}$ appears to be a single, rotationally-broadened standard late-A star and has been widely classified as A7 V, with nearsolar metallicity. There is no hint of change in either the shape or the centroid of the line profiles. The photospheric $\mathrm{K}$ line is strong enough to mask any small, sharp feature which might be present. We confirm the published RV of $-18 \mathrm{~km} \mathrm{~s}^{-1}$, but not the $B S C$ 's qualifying "V".

\section{2. $H R 4875=H D 111604$}

HR 4875 is one of the confirmed $\lambda$ Boo stars in our sample. It appears to be a single, rotationally-broadened late-A star with a metal-deficiency of a factor of $6([\mathrm{M} / \mathrm{H}]=-0.75)$, though we find $[\mathrm{Ca} / \mathrm{Fe}]$ enhanced by $0.1 \mathrm{dex}$ and $[\mathrm{Mg} / \mathrm{Fe}]$ by 0.3 dex. Figure 2 suggests a substantial depression in the continuum near $\lambda 4479 \AA$, as in $\lambda$ Boo and 35 Aql (Fig. 4), though is not quite so clear-cut because its relatively lower $T_{\text {eff }}$ and higher $[\mathrm{M} / \mathrm{H}]$ increase the chance of blending by other lines. As in $21 \mathrm{LMi}$, the photospheric $\mathrm{K}$ line is strong enough to mask any small absorption feature that might also be present. Stütz \& Paunzen (2006) also found no evidence for binarity from their statistical study of photometric indices.

\section{3. $\lambda B O O=H D 125162$}

This sui generis member of the $\lambda$ Boo class appears to be a single, early-A dwarf. Stütz \& Paunzen (2006) also found no evidence of binarity. The metallicity which we derive $([\mathrm{M} / \mathrm{H}]$ $=-2.00,[\mathrm{Ca} / \mathrm{Fe}]=+0.30)$ compares favourably with the value of -2.00 for $[\mathrm{Fe} / \mathrm{H}]$ published by Venn \& Lambert (1990), though those authors derived slightly different $T_{\text {eff }}(8750 \mathrm{~K})$ and $\log g$ (4.0) compared to our values of $8495 \mathrm{~K}$ and 3.90. $\lambda$ Boo exhibits absorption near $\lambda 4479 \AA$ (Fig. 4). There is no detectable K-line pip.

\section{4. $H R 5368=H D 125489$}

HR 5368 appears to be a single, late-A dwarf or sub-giant with slightly enhanced metal abundances of 0.20 dex. Its photospheric $\mathrm{K}$ line is too strong for us to tell whether a sharp absorption pip might be present. CFHT PUEO "adaptive optics" observations in the near-IR (G+2003's Table 3) yielded a positive detection of a companion but with $\Delta m$ of nearly 6 mag, making it "too faint to affect the spectroscopic observations".

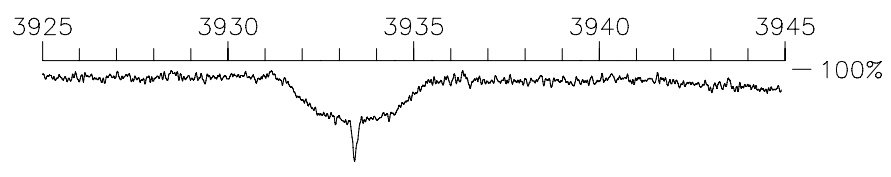

Fig. 7. Enlargement of the Ca II K-line core in 12 Ser. This version, created by averaging the original spectra, has a $S / N$ ratio of about 300 . The narrow absorption is almost certainly interstellar.

\subsection{Ser $=H D 138527$}

12 Ser is rapidly rotating B9 IV star that has been classified as marginal $\lambda$ Boo. Its metals are sub-solar by $0.80 \mathrm{dex}$, and its high projected rotational velocity accentuates the weakness of its lines. It exhibits a prominent K-line pip at a velocity of $-3.8 \mathrm{~km} \mathrm{~s}^{-1}$ relative to the LSR, and since two IS components, at -5.6 and $-2.1 \mathrm{~km} \mathrm{~s}^{-1}$, were observed by Albert et al. (1993) at radio frequencies in the context of mapping the IS gas in the Galactic halo, it seems likely that the K-line pip is IS. An enlarged version of that feature, created as the average of our 5 observations, is shown in Fig. 7.

HIPPARCOS (ESA 1997) detected "duplicity-induced variability" in 12 Ser, but gave no details. G+2003's Table 3 reports the detection of a faint companion $(\Delta m=3.2 \mathrm{mag})$ with the CFHT PUEBO adaptive optics system. We do not know the magnitude difference at visible or blue wavelengths, though if $\Delta m$ is similar in the blue as in the near-IR then the flux from the companion would not be sufficient to veil the lines of the brighter component as has been postulated in order to explain the metal deficiencies which characterize the $\lambda$ Boo stars. Our spectra do not reveal any changes in either the profiles or the positions of lines in the 12 Ser spectrum as would be expected in the case of an an SB2 system (except in the unlikely situation that it was viewed pole-on), nor does its spectrum contain features or line ratios which do not correspond to a synthetic spectrum that is based on solar abundance ratios (see, for instance, Fig. 6b).

\section{6. $H R 6878=H D 169009$}

This object appears to be a single, late-B dwarf with approximately solar overall metallicity, but the profiles and depths of certain lines vary rapidly. Even so, Breger (1969) monitored it for short-term photometric variations and rather surprisingly found none above the $0.001 \mathrm{mag}$ level. Cowley et al. (1969) classified it as B9.5 V “ $\lambda 4005$ enhanced", though Abt \& Morrell (1995) did not see any abundance oddities and classified it as A $1 \mathrm{Vp} \lambda$ Boo. It is included in the Catalogue of Ap, HgMn and Am stars (Renson \& Manfroid 2009), though its peculiar nature is listed as "doubtful".

The line-profile changes which we recorded (see Fig. 8) strongly resemble those in HR 7903 and other CP stars. Chief among them are rapid enhancements in $\mathrm{Cr}$ II, Ti II and Fe II, a general weakness (but to a variable degree) in Ca II (see Fig. 8a) and a marked deficiency in He. One possible explanation is the presence of local aggregations of material with different chemical concentrations, as are often caused by the presence of a strong magnetic field; as such a "spot" is brought into the visible hemisphere through the star's rotation its lines will share the RV of that limb, thereby distorting the averaged profiles. A sequence of spectra can thus be made to yield the rotational velocity of the star. 
A\&A 547, A8 (2012)
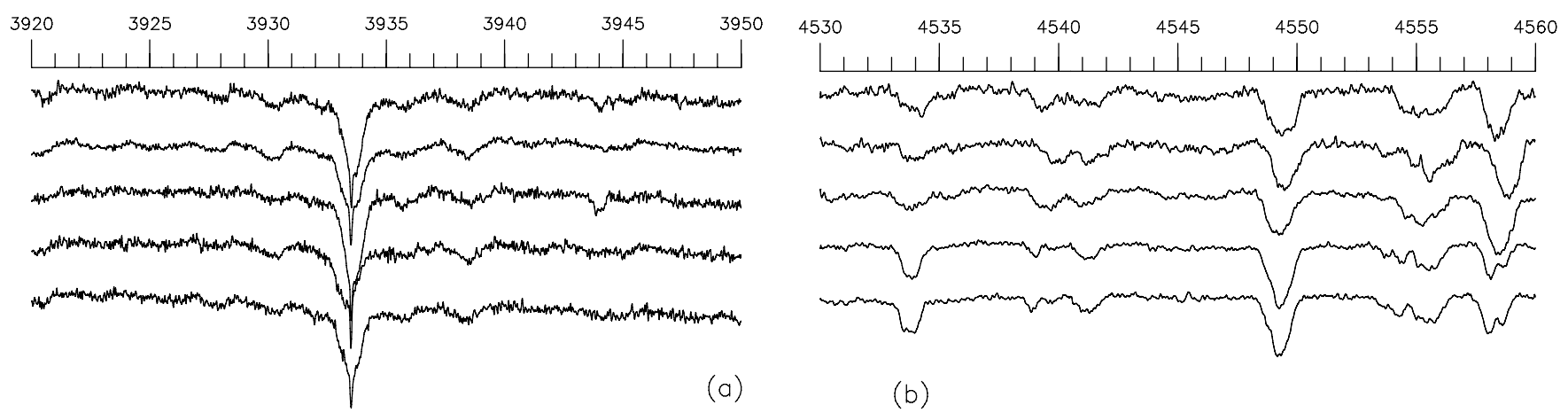

(b)

Fig. 8. Line-profile changes in the UV region a) and in the blue region b) of HR 6878, as revealed by the five respective spectra for those wavelength regions listed in Table 2. In a) the second, third and fourth spectra were observed on consecutive nights, while in b) the first two spectra were separated by an interval of 6 days, and the third and fourth by an interval of 2 days; all the other intervals were substantially longer. The spectra in b) were smoothed by a 9-point function in order to reveal the changes more clearly.

Although a spectral line in a CP star can become asymmetrical, partially split and subsequently re-merge as the spot traverses the disk, the line's broad envelope does not shift appreciably in velocity, and it does not seem reasonable to interpret the line-profile variations and line-depth changes as due to the veiling effects of two similar stars in a composite-binary system; such an explanation has not been proposed or upheld for other CP stars. HR 6878 is to be included in a separate study of the three stars of our sample which proved to have $\mathrm{CP}$ characteristics.

We had averaged our spectra of HR 6878 in order to obtain "representative" ones, and used those to derive the "typical" abundances given in Table 4. The solar value which is recorded there for $[\mathrm{M} / \mathrm{H}]$ was derived from an apparently quiescent spectrum, and may therefore correspond to a least spotted hemisphere. He I at $\lambda 4471 \AA$ is significantly weak.

The $\mathrm{K}$ line contains a strong narrow absorption. Kemp et al. (2002) detected IS features in both the $D$ lines and the $K$ line in this star, and reported the slightly discordant velocities (relative to the LSR) of $+4.5 \pm 1.0 \mathrm{~km} \mathrm{~s}^{-1}$ and $+0.5 \pm 0.5 \mathrm{~km} \mathrm{~s}^{-1}$, which straddle our own value of $+2.1 \mathrm{~km} \mathrm{~s}^{-1}$, and it seems likely that the feature is IS rather than CS. Hempel \& Holweger (2003) included HR 6878 in their careful analysis of element abundances in late B stars, and although they looked for, and found, narrow absorption features in both the $\mathrm{D}$ lines and the $\mathrm{K}$ line in some of their sample they did not record finding any such in HR 6878. We note, too, that they selected HR 6878 because it has relatively slow rotation and is recorded as "normal" in the $B S C$. Accordingly, by observing its spectrum only once, Hempel $\&$ Holweger were unaware of the significant changes in line profiles and apparent abundances which clearly occur during an interval of a few days (see Fig. 8).

HR 6878 appears to have an exceptionally high amount of reddening; our value of $E_{B-V}$ (Table 4) is 5 times greater than for any of the others, though its modest distance of $100 \mathrm{pc}$ is actually the median for the whole set. Our value of $E_{B-V}$ was derived from SIMPLEX fits, and forcing it to be smaller interfered seriously with the overall fit to the photometric data. There is supporting evidence that the high reddening which we found is real. (i) In Table 5 of $\mathrm{G}+2003$, which lists values for $E_{b-y}$, only 5 stars out of the total of 136 have values $>0.1$ mag, and of those HR 6878 ranks third highest with 0.152 ; the equivalent $E_{B-V}$ is $0.21 \mathrm{mag}$. (ii) Scrutiny of the $B S C$ reveals extraordinary reddening in other stars close to the Galactic co-ordinates of HR $6878(l=+21, b=+2)$. (iii) The narrow K-line component, which - as we have already argued - is very likely IS, is easily the strongest example in Fig. 1. Our conclusion is therefore that HR 6878 is indeed highly reddened, and that the parameters we derived for it with SIMPLEX (Table 4) were not compromised.

\section{7. $\lambda A q l=H D 177756$}

The rapid line-profile variations which we detected in this 3 rd-magnitude late-B star have a periodicity of about $15 \mathrm{~h}$. The star's measured rotational velocity is close to $170 \mathrm{~km} \mathrm{~s}^{-1}$, so if it has a radius of (say) $2.75 \pm 0.25 R_{\odot}$ and its rotation axis is inclined at $45 \pm 15^{\circ}$ to the line of sight then its rotation period will also be close to $15 \mathrm{~h}$. Even when the present project was still in its infancy we recognized that the line-profiles of this star changed, but that they did so in ways which did not signal a non-eclipsing SB2 system. Although two distinct, unequal dips appeared in certain lines they subsequently become equal in depth and then merged or swapped places, the envelope profile shifting only a little and altering only slightly in size. We therefore subsequently undertook fairly intense monitoring of $\lambda \mathrm{Aql}$; those investigations, plus additional ones of HR 6878 and HR 7903 as well, will be described in a separate paper.

Despite being such a bright star, $\lambda$ Aql has drawn few comments in the literature. At its spectral type of B8 or B9 its metal lines are naturally weak. It exhibits an approximate overall metal deficiency, $[\mathrm{M} / \mathrm{H}]=-0.5$, and since it is also rotating by $\sim 170 \mathrm{~km} \mathrm{~s}^{-1}$, those lines that are present are very washed out: even the Ca II K line and Mg II $\lambda 4481 \AA$, which are the strongest metal lines in the regions that we observed, are less than $10 \%$ deep. Abt $\&$ Morrell (1995) classified $\lambda$ Aql as B9.5 V p ( $\lambda$ Boo), whereas Cowley et al. (1969) called it B9 Vn "Si", the latter describing a spectrum in which $\mathrm{Si}$ is enhanced but not sufficiently to earn the description "peculiar". He I $\lambda 4471 \AA$ is weaker than expected at the $T_{\text {eff }}$ given for this star in Table 4. Its lines are probably too weak and too blurred by rotation for a meaningful abundance analysis; however, we can report that the Si II lines at $\lambda 4128$ and $4130 \AA$ appear to show solar-ratio abundances. Renson \& Manfroid (2009) included $\lambda$ Aql in their catalogue of CP stars but labelled the peculiar nature of its spectrum as "doubtful".

\section{8. $H R 7288=H D 179791$}

HR 7288 appears to be an unexceptional, single, A1.5 IV star, with a substantial rotational velocity of about $200 \mathrm{~km} \mathrm{~s}^{-1}$. Our measurement of its RV is $+13 \mathrm{~km} \mathrm{~s}^{-1}$, rather than the published value of $+9 \mathrm{~km} \mathrm{~s}^{-1}$, and we do not agree that the velocity is variable as if it had a companion of stellar mass. We find no evidence 
R. E. Griffin et al.: The $\lambda$ Boo class of stars
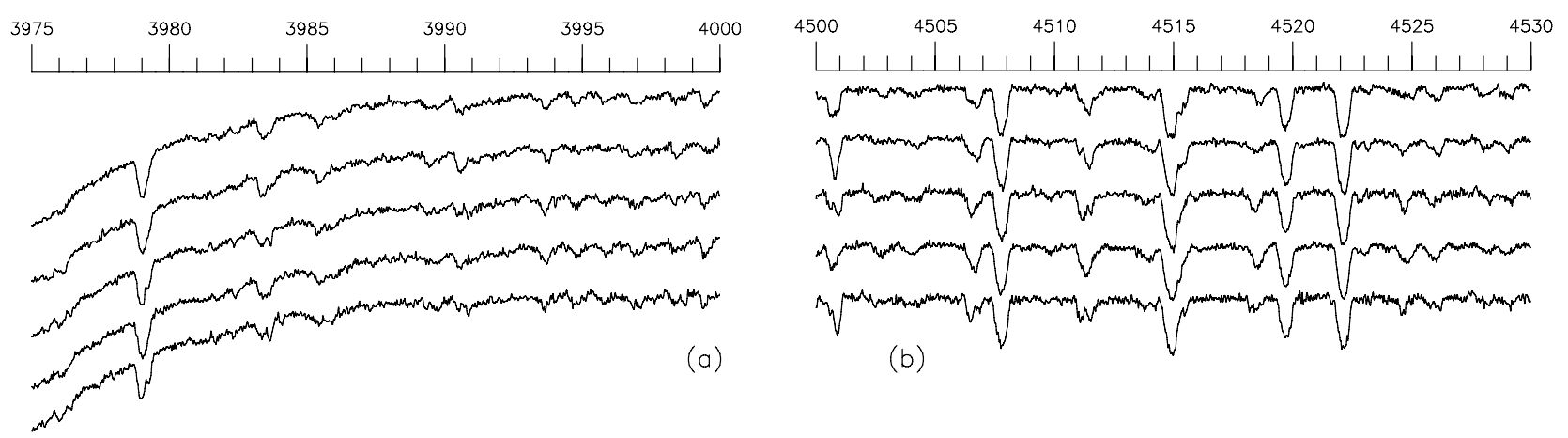

Fig. 9. Line-profile changes in the UV region a) and in the blue b) in of HR 7903, as revealed by the five spectra for those respective wavelength regions listed in Table 2. In a) the second and third spectra were separated by a gap of 2 nights, while in b) the second, third and fourth spectra were taken on successive nights; all the other intervals represented here were substantially longer.

that its spectrum is composite. We detected a small K-line pip at a velocity of $-1.0 \mathrm{~km} \mathrm{~s}^{-1}$ relative to the LSR; that value is close to an IS feature at $-1.2 \mathrm{~km} \mathrm{~s}^{-1}$ in this star's $\mathrm{Na} \mathrm{D}$ lines reported by Génova et al. (1997) and strongly suggests that the K-line pip is IS rather than CS. HR 7288 is included in the CP catalogue of Renson \& Manfroid (2009) but labelled "doubtful"; we could not track down what prompted that inclusion.

\section{9. $35 \mathrm{Aql}=H D 183324$}

Widely recognized as one of the "classical" $\lambda$ Boo stars, 35 Aql appears to be a single object with a modest projected rotation of $\sim 100 \mathrm{~km} \mathrm{~s}^{-1}$ and a general metal deficiency of $-1.8 \mathrm{dex}$ (but $[\mathrm{Ca} / \mathrm{Fe}]=+0.4)$. A persistent asymmetry in the $\mathrm{K}$ line at a blueshifted velocity of over $30 \mathrm{~km} \mathrm{~s}^{-1}$, and nearly $-50 \mathrm{~km} \mathrm{~s}^{-1}$ relative to the star itself, may also be present in the Na I D lines according to Fig. 7 of Bohlender et al. (1999), and may be related to the non-radial pulsations which that star undergoes. A velocity of that magnitude does not fit comfortably with observations of the IS medium; a survey by Génova et al. (1997) of IS Na I in a star close to the same line of sight records a velocity relative to the LSR of $-1.8 \mathrm{~km} \mathrm{~s}^{-1}$, so the origin of the pip is likely to be CS. $35 \mathrm{Aql}$ also shows evidence of $I R$ excess, implying the presence of a CS debris disk. Our RV measurement for the star itself is well defined, and agrees with that given in the $B S C$ to within its precision. We find no evidence that the spectrum is composite, nor did Stütz \& Paunzen (2006).

\subsection{0. $H R 7903=H D 196821$}

There is general consensus in the recent literature that HR 7903 is a CP star of the HgMn type, though Cowley et al. (1969) gave A0 III, "Ca extremely weak", while AM95 preferred A0 IIIp ( $\lambda$ Boo). Renson \& Manfroid (2009) offered "Ap Cr?" for it in their catalogue of CP stars. Changes in the profiles and depths of lines in HR 7903 are unmistakeable (see Fig. 9) and - as for HR 6878 - the overall envelope of a line does not move, thus suggesting a variable abundance pattern on the stellar surface rather than an SB2 spectrum. HR 7903 shows a weak feature at the wavelength of $\mathrm{Hg}$ II $\lambda 3983.9 \AA$, whose variable profile matches those of other enhanced elements in this star. It also has a noticeable deficiency in He. HR 7903 and HR 6878 actually resemble one another far more closely than does either star to the corresponding synthetic spectrum calculated with solarabundance ratios. Our best-fitting model for HR 7903 indicates substantial variations from the solar-abundance ratios, though we adopted $[\mathrm{M} / \mathrm{H}]=0.00$ as a compromise for spectrum fitting when the prime purpose was to match the Balmer lines. While
Fe has an abundance close to the solar one, $\mathrm{Cr}$ and $\mathrm{Mn}$ are both enhanced by more than a factor of 10 but $\mathrm{Ti}$ and $\mathrm{Mg}$ are deficient by factors of 3 or 4 . Fitting to the $\mathrm{K}$ line indicated a significant deficiency in $\mathrm{Ca}$ of at least 1.0 dex, not allowing for a nonnegligible IS contribution to that line.

HR 7903 shows a sharp and fairly strong, though blended, absorption feature in the K line. Génova et al. (1997) measured the velocity of an IS feature in the $\mathrm{Na} \mathrm{D}$ lines in a star in the same direction as $+3.1 \mathrm{~km} \mathrm{~s}^{-1}$, which is close enough to the value of $+2.7 \mathrm{~km} \mathrm{~s}^{-1}$ given in Table 5 for us to identify the narrow K-line absorption in HR 7903 as IS.

Notwithstanding the opinion of an expert classifier (Bidelman 1988) that its "odd" spectrum resembled an Ap Cr star, F+2004 "confirmed" that it is SB because in their one spectrum, of the O I triplet, the three lines "all display the same kind of asymmetry". Our own numerous observations of the star suggest that their bold confirmation was an ill-founded leap of faith.

\subsection{1. $\tau$ Peg $=H D 220061$}

This A8 dwarf appears to be a single star, though it was found by HIPPARCos to show small photometric variations, of order 0.03 mag. with a period of 0.054 days. It has been tentatively classified as $\lambda$ Boo, but has only a rather mild metal deficiency of a factor of 2 . The RV of $+6.1 \mathrm{~km} \mathrm{~s}^{-1}$ which we determined is significantly different from the mean value of $+16 \mathrm{~km} \mathrm{~s}^{-1}$ quoted in the $B S C$. From the literature SIMBAD retrieves 8 measurements, of which one is highly uncertain and one (a negative value) refers to HR 8800, not HR 8880 ( $\tau$ Peg), and should be expunged. The remaining 6 values cluster round the $B S C$ mean. However, there is no hint in either Figs. 1 or 2 of any line-profile variations which would have to be visible if the star were a binary with a variable RV component in the line of sight. Erspamer \& North (2003) also analysed spectra of $\tau$ Peg, finding $T_{\text {eff }}=7655 \mathrm{~K}$, $\log g=3.78, v \sin i=154 \mathrm{~km} \mathrm{~s}^{-1}$ and $[\mathrm{Fe} / \mathrm{H}]=-0.28-$ none very different from the values in Table 4 . There is also no hint of a sharp K-line feature, but the star is too close $(50 \mathrm{pc})$ for significant IS contribution.

\subsection{And $=H D 221756$}

Another widely-recognized classical $\lambda$ Boo star, 15 And appears to be single, with a metal deficiency of a factor of 6 . It shows a small blue-shifted pip in the Ca II $\mathrm{K}$ line, and like $35 \mathrm{Aql}$ - it exhibits an $I R$ excess that could be due to a $\mathrm{CS}$ debris disk. It is one of the $\lambda$ Boo stars in which Bohlender et al. (1999) discovered nonradial pulsations. According to our measurements, its RV appears to be constant to well within the 
precisions quoted in Table 3 , and the mean is also consonant with the $+13 \mathrm{~km} \mathrm{~s}^{-1}$ quoted elsewhere. We find no evidence that the spectrum could be SB2; Stütz \& Paunzen (2006) came to a similar conclusion.

\section{Conclusions and comments}

$\mathrm{G}+2003, \mathrm{~F}+2004$ and previous papers by the same group have claimed persistently that $\lambda$ Boo stars do not exist as a class, and that their spectrum peculiarities can be explained away as some form of duplicity. Out of a subset of 14 which were listed as " $\lambda$ Boo candidates" and singled out as "likely [to] display composite spectra", we have exhaustively monitored the spectra of 10 of them at two different wavelengths, and added 2 more - one being $\lambda$ Boo itself, the other being another star from the G+2003 list which F+2004 argued is a SB2 system. We found no evidence at all for SB2 systems. None of the spectra shows any hint of a close binary companion. We therefore do not agree that the $\lambda$ Boo phenomenon can be explained away by the hypothesis that such stars are actually composite-spectrum binaries. The final conclusion of $\mathrm{G}+2003$ asserts that "it is very easy to classify a binary as a $\lambda$ Boo star". We have in fact found it impossible to confirm that explanation for a sub-set of 12 stars explicitly singled out by those authors as the most likely candidates. By adopting the same sub-set of stars which were strongly suggested by F+2004 from statistical arguments as being composite-spectrum binaries, and by not introducing any spectral-type selection or grouping of our own, we have been able to confront the composite-spectrum binary hypothesis at the same level as that at which it was conceived.

The arguments put forward by G+2003 and F+2004, that the weakness of lines in $\lambda$ Boo stars is due to infilling of one star's lines by the continuum flux of its companion, require a velocity separation of sufficient magnitude so as to resolve their individual lines at nodal phases. If we exclude the two CP stars HR 6878 and HR 7903 (whose rotation is more modest) the average projected rotation rate of the rest is nearly $150 \mathrm{~km} \mathrm{~s}^{-1}$. Orbital amplitudes of $300 \mathrm{~km} \mathrm{~s}^{-1}$ or more would therefore be required on average to bring about line-weaking by displacing one spectrum relative to the other. Even so, one cannot explain a metal deficiency of 1 to 2 dex by that mechanism.

In contrast to the above assertion as to what the $\lambda$ Boo stars are not, this paper is less confident about stating what they are. Table 4 indicates that the 6 confirmed or probable $\lambda$ Boo stars in our sample are substantially metal-poor as a group, but that their average rotational velocity of $125 \mathrm{~km} \mathrm{~s}^{-1}$ is hardly different from the $117 \mathrm{~km} \mathrm{~s}^{-1}$ which characterizes the rest of the sample. There may also be a tendency for $[\mathrm{Ca} / \mathrm{Fe}]$ and $[\mathrm{Mg} / \mathrm{Fe}]$ to be a bit raised, and thus non-solar, but that does not appear to be a general property. Half of the $\lambda$ Boo stars in the sample exhibit a narrow absorption feature in the $\mathrm{Ca}$ II $\mathrm{K}$ line which (it has been suggested) could originate in the CS disk that was or is also responsible for contaminating each star's surface, but equally half do not, and in at least one of the positive cases (12 Ser) it is likely to be interstellar in origin rather than circumstellar. However, if - as some authors argue - the narrow feature is only seen when the star's axis of rotation is such that the disk is viewed nearly edge-on, it would not be visible in every case anyway. There is some evidence for a broad absorption feature near $\lambda 4479 \AA$ in the $\lambda$ Boo stars; only in 12 Ser does it definitely seem to be absent, though 12 Ser is also considerably hotter than the other $5 \lambda$ Boo stars.

Although it has not been the aim of this paper to study whichever mechanism or evolutionary state may be responsible for this somewhat tantalizing group, we are decided in our assertion that they are a distinct group of chemically-peculiar stars and that they cannot be explained away by suggestions of an unresolved or undetected composite nature. The expert eyes of numerous past classifiers have been able to recognize each upon sight, and we have found no reason so far to doubt their conclusions.

Our study has incidentally brought to light the discovery of CP-like variations in a third-magnitude star that have apparently escaped mention to date, and has discovered or confirmed the CP nature of two others in the sample of 12. A detailed study of those three CP candidates is being prepared.

We have emphasized the importance of ascertaining carefully whether a parameter is likely to be truly variable before announcing it in print. Much of the work entailed in the present study could have been avoided had the $B S C$ not attributed variable RV to all the early-type stars whose individual values disagreed by amounts which were in fact not surprising given the difficulties of measuring such quantities at low dispersion in stars which show only rotationally-broadened Balmer lines. A case of "confirmed" SB2 was also reported by F+2004 on the basis of one spectrum whose lines showed asymmetry, necessitating numerous observations in order to check (and actually refute) that claim.

Acknowledgements. R.E.M.G. thanks the Herzberg Institute of Astrophysics in Victoria for its hospitality, and in particular for abundant observing time on the $1.2-\mathrm{m}$ telescope where all the spectra referred to above were obtained.

\section{References}

Abt, H. A., \& Morrell, N. I. 1995, ApJS, 99, 135

Albert, C. E., Blades, J. C., Morton, D. C., et al. 1993, ApJS, 88, 81

Andersen, J. 1991, A\&ARv, 3, 91

Bidelman, W. P. 1988, PASP, 100, 1084

Bohlender, D. A., Gonzalez, J.-F., \& Matthews, J. M. 1999, A\&A, 350, 553

Breger, M. 1969, ApJS, 19, 79

Castelli, F., \& Kurucz, R. L. 2003, in Modelling of Stellar Atmospheres, eds. N. E. Piskunov, W. W. Weiss, \& D. F. Gray (San Francisco: ASP), A20

Cowley, A., Cowley, C., Jaschek, M., \& Jaschek, C. 1969, AJ, 74, 375

Erspamer, D., \& North, P. 2003, A\&A, 398, 1121

ESA 1997, The HipParcos and Tycho Catalogues, ESA Publ. Division, Noordwijk, ESA SP, 1200

Evans, D. S. E. 1967, Revision of the General Catalogue of Radial Velocities, IAUS, 30, 57

Faraggiana, R., \& Bonifacio, P. 1999, A\&A, 349, 521

Faraggiana, R., Bonifacio, P., Caffau, E., Gerbaldi, M., \& Nonino, M. 2004, A\&A, 425, $615(\mathrm{~F}+2004)$

Francis, C., \& Anderson, E. 2009, J. New Ast., 14, 615

Genova, R., Beckman, J. E., Bowyer, S., \& Spicer, T. 1997, ApJ, 484, 761

Gerbaldi, M., Faraggiana, R., \& Lai, O. 2003, A\&A, 412, 447 (G+2003)

Gray, R. O., \& Corbally, C. J. 1994, AJ, 107, 742

Gray, R. O., Graham, P. W., \& Hoyt, S. R. 2001, AJ, 121, 2159

Gray, R. O., Corbally, C. J., Garrison, R. F., McFadden, M. T., \& Robinson, P. E. 2003, AJ, 126, 2048

Hoffleit, D. 1982, Bright Star Catalogue, 4th edn. (New Haven: Yale Univ. Obs.) Hempel, M., \& Holweger, H. 2003, A\&A, 408, 1065

Holweger, H., \& Rentzsch-Holm, I. 1995, A\&A, 303, 819

Holweger, H., Hempel, M., \& Kamp, I. 1999, A\&A, 250, 603

Kemp, S. N., Bates, B., Beckman, J. E., et al. 2002, MNRAS, 333, 561

Paunzen, E. 2001, A\&A, 373, 633

Pols, O. R., Schröder, K.-P., Hurley, J. R., Tout, C. A., \& Eggleton, P. P. 1998, MNRAS, 298, 525

Renson, P., \& Manfroid, J. 2009, A\&A, 498, 961

Stütz, C., \& Paunzen, E. 2006, A\&A, 458, L17

Su, K. Y. L., Rieke, G. H., Stansberry, J. A., et al. 2006, ApJ, 653, 675

Thompson, G. I., Nandy, K., Jamar, C., et al. 1978, Catalogue of Stellar Ultraviolet Fluxes (London: Sci. Res. Council)

van Leeuwen, F. 2007, A\&A, 474, 653

Venn, K. A., \& Lambert, D. L. 1990, ApJ, 363, 234

Wilson, R. E. 1953, General Catalog of Stellar Radial Velocities, CIW, Washington, DC 